QUARTERLY OF APPLIED MATHEMATICS

VOLUME LXVIII, NUMBER 4

DECEMBER 2010, PAGES 607-643

S 0033-569X(2010)01167-7

Article electronically published on September 17, 2010

\title{
TWO-DIMENSIONAL REACTION-DIFFUSION EQUATIONS WITH MEMORY
}

\author{
BY \\ MONICA CONTI (Dipartimento di Matematica "F.Brioschi", Politecnico di Milano Via Bonardi 9, \\ 20133 Milano, Italy), \\ STEFANIA GATTI (Dipartimento di Matematica, Università di Modena e Reggio Emilia via Campi \\ 213/B, 41100 Modena, Italy), \\ MAURIZIO GRASSELLI (Dipartimento di Matematica "F.Brioschi", Politecnico di Milano Via \\ Bonardi 9, 20133 Milano, Italy), \\ AND \\ VITTORINO PATA (Dipartimento di Matematica "F.Brioschi", Politecnico di Milano Via Bonardi \\ 9, 20133 Milano, Italy)
}

\begin{abstract}
In a two-dimensional space domain, we consider a reaction-diffusion equation whose diffusion term is a time convolution of the Laplace operator against a nonincreasing summable memory kernel $k$. This equation models several phenomena arising from many different areas. After rescaling $k$ by a relaxation time $\varepsilon>0$, we formulate a Cauchy-Dirichlet problem, which is rigorously translated into a similar problem for a semilinear hyperbolic integro-differential equation with nonlinear damping, for a particular choice of the initial data. Using the past history approach, we show that the hyperbolic equation generates a dynamical system which is dissipative provided that $\varepsilon$ is small enough, namely, when the equation is sufficiently "close" to the standard reactiondiffusion equation formally obtained by replacing $k$ with the Dirac mass at 0 . Then, we provide an estimate of the difference between $\varepsilon$-trajectories and 0 -trajectories, and we construct a family of regular exponential attractors which is robust with respect to the singular limit $\varepsilon \rightarrow 0$. In particular, this yields the existence of a regular global attractor
\end{abstract}

Received January 13, 2009.

2000 Mathematics Subject Classification. Primary 45K05; Secondary 35B40, 35B41, 76A10, 92D25.

Key words and phrases. Reaction-diffusion equations, memory effects, nonlinear damping, exponential attractors, global attractors, Lyapunov functionals, convergence to equilibria.

This work was partially supported by the Italian PRIN Research Project 2006 Problemi a frontiera libera, transizioni di fase e modelli di isteresi.

E-mail address: monica.conti@polimi.it

E-mail address: stefania.gatti@unimore.it

E-mail address: maurizio.grasselli@polimi.it

E-mail address: vittorino.pata@polimi.it

(C)2010 Brown University Reverts to public domain 28 years from publication 
of finite fractal dimension. Convergence to equilibria is also examined. Finally, all the results are reinterpreted within the original framework.

\section{Contents}

\begin{tabular}{l|l|} 
1. Introduction & 608 \\
2. Notation and basic assumptions & 611 \\
3. Statements of the main results & 612 \\
4. The continuous dependence estimate & 614 \\
5. An equivalent problem & 615 \\
6. A dynamical system in the history space framework & 621 \\
7. Dissipativity & 622 \\
8. Higher-order dissipativity & 626 \\
9. Continuous dependence for smoother initial data & 629 \\
10. Exponential asymptotic smoothing & 633 \\
11. Estimate of the difference of trajectories & 636 \\
12. Robust exponential attractors & 640 \\
13. Global attractors and convergence to equilibria & 642 \\
14. Conclusion: Proofs of the main results \\
References
\end{tabular}

1. Introduction. Let $\Omega \subset \mathbb{R}^{N}$ be a bounded domain with smooth boundary $\partial \Omega$. A very well-known equation ruling the evolution of a function $u: \Omega \times[0, \infty) \rightarrow \mathbb{R}$ is the semilinear parabolic equation

$$
\partial_{t} u-\Delta u+\phi(u)=0
$$

where $\phi$ is a nonlinear smooth function whose typical form is an odd polynomial with positive leading coefficient. This equation, which accounts for diffusion and reaction effects, serves as a model for several different phenomena arising, for instance, from Physics, Chemistry, and Biology. Its mathematical properties have been widely investigated in previous decades by a large number of authors who proved, in particular, several results about the asymptotic behavior of solutions (e.g., [2, 4, 27, 30, 31, 40]). On the other hand, from the physical viewpoint, equation (1.1) presents somehow a major flaw since, due to its purely parabolic character, it predicts an infinite speed of propagation of disturbances, which obviously cannot occur in the real phenomena. A reasonable and physically meaningful way to avoid this unpleasant feature is to assume a delay mechanism preventing instantaneous regularization effects, meaning that the actual value of $u(t)$ is influenced by its past history $u(\tau), \tau<t$, which produces an inertial effect. Along this line, a quite interesting and mathematically challenging modification of (1.1) is the reaction-diffusion equation with memory

$$
\partial_{t} u-\int_{0}^{t} k(s) \Delta u(t-s) d s+\phi(u)=0
$$

where $k:[0, \infty) \rightarrow[0, \infty)$ is a nonincreasing summable kernel. This equation is hyperbolic in the sense that the energy of a initial given perturbation in a bounded subset of $\Omega$ propagates with finite speed (see [16] and references therein). In particular, in the simple, albeit basic, case

$$
k(t)=\frac{1}{\varepsilon} e^{-\frac{t}{\varepsilon}},
$$


for some given $\varepsilon \in(0,1]$, called relaxation time, equation (1.2) can be formally transformed into the hyperbolic equation

$$
\varepsilon \partial_{t t} u-\Delta u+\left(1+\varepsilon \phi^{\prime}(u)\right) \partial_{t} u+\phi(u)=0,
$$

which, provided that $\phi^{\prime}$ is bounded from below, becomes dissipative for small values of $\varepsilon$. However, if $k$ reduces to the Dirac mass at 0 (i.e., the past memory is lost), we recover (1.1). This suggests that (1.1) is a good approximation of (1.2) when the system keeps a very short memory. If so, then it is reasonable to work with (1.1) in place of (1.2), with the great advantage of handling a much simpler equation.

Concerning concrete applications, (1.2) has been proposed as a model of viscoelastic fluids [8, 11, 35. Alternatively, it can be viewed as a semilinear (hyperbolic) heat equation based on the Gurtin-Pipkin heat conduction law [18, 22, 34, or as a simple model for chemosensitive movements [28, 29]. Its relevance has been further underlined in a series of papers (see [10, 32, 33] and references therein). In those contributions, the authors refer to (1.3) as a hyperbolic reaction-diffusion equation, and they show how it can be used to describe a number of phenomena of biological and chemical interest, such as chemically reacting systems, gene selection, population dynamics, and forest fire propagation. Despite these attractive features, very few rigorous mathematical results are available so far. Regarding existence and uniqueness, some theorems can be found in [1, where (1.2) is incorporated in a phase-field system and $\phi$ is allowed to be a maximal monotone graph. In [6, 18, the infinite time delay version of (1.2) is shown to generate a dynamical system in the history space framework. In particular, considering the rescaling $k_{\varepsilon}(s)=\varepsilon^{-1} k(s / \varepsilon)$ of the original kernel $k$, with a small parameter $\varepsilon>0$, the paper [6] establishes the convergence on finite time intervals of the solutions to (1.2) to the solutions to (1.1) in the limit $\varepsilon \rightarrow 0$, provided that the initial data are regular enough. Moreover, in the one-dimensional case, 21] proves the existence of the global attractor for small values of $\varepsilon$, exploiting the precompact pseudometric technique [23, but without any regularity result. We remark that, in [6, 21, the phase space for $u$ is $L^{2}(\Omega)$. In this functional setting, it seems particularly hard to find satisfactory global asymptotic results, such as the existence of regular global attractors or of exponential attractors. Nonetheless, if we work in the smaller phase space $H^{1}(\Omega)$, as [18] does, then we can appeal to a more familiar hyperbolic formulation, close to (1.3). In which case, a small relaxation time $\varepsilon$ is needed in order to have dissipativity. This is motivated by the recent papers [15, 36, 37, focused on (1.3) in different space dimensions, with $1+\varepsilon \phi^{\prime}(u)$ replaced by a generic damping coefficient $\sigma(u) \geq \sigma_{0}>0$. These papers shed a new light on the approach devised in [18, based on the reformulation of equation (1.2) as a hyperbolic equation similar to (1.3), but also containing a convolution term.

The present work is devoted to a detailed investigation of the two-dimensional case, which is of particular interest for biological and chemical applications. The main goal is the analysis of the longtime behavior of solutions, with special regard to their dependence on $\varepsilon$, in the spirit of $[6,9,14,24$. More precisely, we compare in a quantitative way the closeness of the global dynamics of (1.2) and (1.1). As a consequence, it will be clear that the latter equation can be viewed as a good approximation of (1.2) when $k$ is rapidly fading (short memory), not only on finite time intervals, but also asymptotically. In this 
framework, we can no longer speak in terms of dynamical systems. Thus, we need to rephrase some notions like, for instance, the one of exponential attractor. Nonetheless, the past history approach, which allows the use of the semigroup language, is hidden in the proofs, and all the results can be formulated for the infinite delay version of (1.2) as well. Finally, we remark that the presence of an extra source term (even time-dependent) in the equation can be handled without significant changes in the proofs.

We can now enter the details. Let $\Omega \subset \mathbb{R}^{2}$ be a bounded domain with smooth boundary $\partial \Omega$; then, for $t>0$ and $\varepsilon \in(0,1]$, we consider the following integro-differential equation of Volterra type for the unknown variable $u=u(\boldsymbol{x}, t): \Omega \times[0, \infty) \rightarrow \mathbb{R}$ :

$$
\partial_{t} u(t)-\int_{0}^{t} k_{\varepsilon}(s) \Delta u(t-s) d s+\phi(u(t))=0,
$$

supplemented with the initial and boundary value conditions

$$
\left\{\begin{array}{l}
u(\boldsymbol{x}, 0)=u_{0}(\boldsymbol{x}), \quad \boldsymbol{x} \in \Omega, \\
u(\boldsymbol{x}, t)=0, \quad \boldsymbol{x} \in \partial \Omega, t \geq 0,
\end{array}\right.
$$

where $u_{0}$ is assumed to be known, and

$$
k_{\varepsilon}(s)=\frac{1}{\varepsilon} k\left(\frac{s}{\varepsilon}\right)
$$

is the rescaling of a nonincreasing and summable memory kernel $k:[0, \infty) \rightarrow[0, \infty)$, subject to the normalization condition

$$
\int_{0}^{\infty} k(s) d s=1
$$

We also assume that $k^{\prime}$ is summable and absolutely continuous on $\mathbb{R}^{+}=(0, \infty)$, and satisfies the differential inequality

$$
k^{\prime \prime}(s)+\alpha k^{\prime}(s) \geq 0
$$

for some $\alpha>0$ and almost every $s>0$. This includes in the picture, among others, exponential kernels. In the (singular) limit $\varepsilon \rightarrow 0$, the kernel $k_{\varepsilon}$ converges in the sense of distribution to the Dirac mass at 0. Accordingly, (1.4) formally reduces to (1.1).

Plan of the paper. The next section essentially contains the basic assumptions on the nonlinearity $\phi$. Section 3 contains the main results stated for the Volterra equation (1.4) endowed with the initial and boundary value conditions (1.5). In particular, Theorem 3.7 is concerned with the existence of a family of sets with finite fractal dimension which are compact in $H_{0}^{1}(\Omega)$ and attract exponentially fast any set of initial data bounded in $H_{0}^{1}(\Omega)$. Moreover, the Hausdorff distance between such sets and a suitable exponential attractor of the corresponding limiting equation (1.1) can be controlled by a constant times a certain power of $\varepsilon$. A useful continuous dependence estimate is proved in Section 4, while Section 5 is devoted to establishing the equivalence of (1.4) (1.5) with a hyperbolic integro-differential equation similar to (1.3). The latter equation is analyzed in Section 6, where, for any $\varepsilon \in(0,1]$, it is shown to generate a semigroup $S_{\varepsilon}(t)$, acting on the extended phase space which accounts for the past history. Sections 7 and 8 are concerned with the dissipativity properties of $S_{\varepsilon}(t)$ when $\varepsilon$ is small enough, i.e., the existence 
of bounded absorbing sets. A particularly useful continuous dependence estimate is obtained in Section 9, but the crucial properties are demonstrated in Section 10 (existence of a compact exponentially attracting set) and in Section 11 (estimate of the difference between $\varepsilon$-trajectories and 0 -trajectories). In Section 12, we collect all the previous results and prove the existence of a family of exponential attractors which is robust as $\varepsilon$ goes to 0 . Section 13 is dedicated to some properties of the global attractor and to the convergence to single equilibria via the Łojasiewicz-Simon inequality. Finally, in Section 14 , we rephrase all the main theorems obtained in the history space setting in terms of the original problem (1.4)-(1.5).

2. Notation and basic assumptions. Given a Banach space $X$, we denote by $\boldsymbol{\delta}_{X}$ and $\boldsymbol{\delta}_{X}^{\text {sym }}$ the usual Hausdorff semidistance and the symmetric Hausdorff distance in $X$, respectively.

Setting $\left(H^{0},\|\cdot\|,\langle\cdot, \cdot\rangle\right)=L^{2}(\Omega)$, we consider the selfadjoint operator

$$
-\Delta: H^{2}(\Omega) \cap H_{0}^{1}(\Omega) \subset H^{0} \rightarrow H^{0} .
$$

Then, for every $r \in \mathbb{R}$, we define the scale of Hilbert spaces

$$
H^{r}=\operatorname{dom}\left((-\Delta)^{\frac{r}{2}}\right), \quad\left\langle u_{1}, u_{2}\right\rangle_{H^{r}}=\left\langle(-\Delta)^{\frac{r}{2}} u_{1},(-\Delta)^{\frac{r}{2}} u_{2}\right\rangle .
$$

In particular,

$$
H^{-1}=H^{-1}(\Omega), \quad H^{1}=H_{0}^{1}(\Omega), \quad H^{2}=H^{2}(\Omega) \cap H_{0}^{1}(\Omega) .
$$

The symbol $\langle\cdot, \cdot\rangle$ will also be used for duality.

Being in space dimension two, we will often exploit the continuous embeddings

$$
H^{1} \subset L^{p}(\Omega), \quad p \geq 1,
$$

as well as the Gagliardo-Nirenberg inequality

$$
\|u\|_{L^{2 p}} \leq C\|u\|^{\frac{1}{p}}\|\nabla u\|^{1-\frac{1}{p}}, \quad p \geq 1 .
$$

Throughout the paper, the symbols $C$ and $\mathcal{Q}$ will stand for a generic constant and a generic positive increasing function, respectively, both independent of $\varepsilon$.

Assumptions on $\phi$. Let $\phi \in C^{3}(\mathbb{R})$, with $\phi(0)=0$, be such that

$$
\liminf _{|u| \rightarrow \infty} \frac{\phi(u)}{u}>-\lambda_{1},
$$

where $\lambda_{1}$ is the first eigenvalue of $-\Delta$, and

$$
\phi^{\prime}(u) \geq-\ell,
$$

for some $\ell \geq 0$. We also require that there exist $L>\ell$ and $\gamma \in(0,1]$ such that

$$
\left|\phi^{\prime \prime}(u)\right| \leq C\left[L+\phi^{\prime}(u)\right]^{1-\gamma} .
$$

In particular, (2.4) implies that

$$
\left|\phi^{\prime}(u)\right| \leq C\left(1+|u|^{\frac{1}{\gamma}}\right) .
$$

We can immediately assert that any odd polynomial vanishing at zero with positive leading coefficient is an allowed nonlinearity. This includes the physically significant case of the derivative of the double-well potential, namely, $\phi(u)=u^{3}-u$. 
3. Statements of the main results. We first stipulate the definition of a (weak) solution to (1.4)- (1.5)

Definition 3.1. A function

$$
u \in L^{\infty}\left([0, T], H^{1}\right)
$$

is a solution to (1.4) - (1.5) on the time interval $[0, T]$ if $u(0)=u_{0}$ and, for every $t \in(0, T]$ and every test function $w \in H^{1}$,

$$
\left\langle\partial_{t} u(t), w\right\rangle+\int_{0}^{t} k_{\varepsilon}(s)\langle\nabla u(t-s), \nabla w\rangle d s+\langle\phi(u(t)), w\rangle=0 .
$$

Note that, by comparison, $\partial_{t} u \in L^{\infty}\left([0, T], H^{-1}\right)$. Hence, $u \in C\left([0, T], H^{0}\right)$, so that the initial condition is well defined.

Proposition 3.2. For any $T>0$ and any $\varepsilon \in(0,1]$, problem (1.4)-(1.5) has a unique solution $u$ on $[0, T]$. Moreover,

$$
\partial_{t} u \in L^{\infty}\left([0, T], H^{0}\right)
$$

Due to this last control and the polynomial growth of $\phi$, we learn from (1.4) that

$$
\int_{0}^{t} k_{\varepsilon}(s) \Delta u(t-s) d s \in L^{\infty}\left([0, T], H^{0}\right) .
$$

As a consequence, we obtain the following straightforward corollary.

Corollary 3.3. A weak solution to (1.4)-(1.5) is also strong. Namely, it solves (1.4) almost everywhere.

The existence and uniqueness (in fact, continuous dependence) result in $H^{1}$ is wellknown to hold also for the limiting situation corresponding to $\varepsilon=0$, that is, for equation (1.1) with initial and boundary conditions (1.5). In that case, however, one has less regularity for $\partial_{t} u$, namely, $\partial_{t} u \in L^{\infty}\left([0, T], H^{-1}\right)$.

In light of these facts, for every $\varepsilon \in[0,1]$, we introduce the one-parameter family of operators

$$
U_{\varepsilon}(t): H^{1} \rightarrow H^{1}
$$

defined as

$$
U_{\varepsilon}(t) u_{0}=u(t),
$$

where $u(t)$ is the unique solution at time $t$ to (1.4) (to (1.1) if $\varepsilon=0$ ), with initial and boundary conditions (1.5). Note that, except in the limiting case $\varepsilon=0$, where $U_{0}(t)$ is a (strongly continuous) semigroup on $H^{1}$, the family $U_{\varepsilon}(t)$ is not a semigroup, due to the presence of the convolution integral. However, we can state a weaker continuous dependence result which holds for all $\varepsilon \in[0,1]$.

Proposition 3.4. For every $\varepsilon \in[0,1]$ and every $t \in[0, T]$, we have the estimate

$$
\left\|U_{\varepsilon}(t) u_{1}-U_{\varepsilon}(t) u_{2}\right\| \leq \mathcal{Q}(T)\left\|u_{1}-u_{2}\right\| .
$$

The next theorem provides an estimate of the closeness of the trajectories of (1.4) and (1.1), originating from the same (smoother) initial data, on finite time intervals. 
Theorem 3.5. Let $a<1 / 4$ be fixed. Then, for every $\varepsilon \in(0,1], t \in[0, T], R>0$ and $u_{0} \in H^{2}$ such that

$$
\left\|\Delta u_{0}\right\| \leq R
$$

it follows that

$$
\left\|\nabla U_{\varepsilon}(t) u_{0}-\nabla U_{0}(t) u_{0}\right\| \leq \mathcal{Q}(T+R) \varepsilon^{a} .
$$

If $\varepsilon$ is small enough, the long-term dynamics are confined in a bounded set, uniformly with respect to $\varepsilon$. Precisely, fixing

$$
\varepsilon_{0} \in(0,1] \text { such that } \alpha-\varepsilon_{0} \ell>0,
$$

we have

Theorem 3.6. There exists a bounded set $\mathcal{B}^{0} \subset H^{1}$ satisfying the following properties: for every $\varepsilon \in\left[0, \varepsilon_{0}\right]$ and every bounded set $\mathcal{B} \subset H^{1}$, there exists $t_{0}=t_{0}\left(\|\mathcal{B}\|_{H^{1}}\right) \geq 0$, independent of $\varepsilon$, such that

$$
U_{\varepsilon}(t) \mathcal{B} \subset \mathcal{B}^{0}, \quad \forall t \geq t_{0}
$$

Moreover,

$$
U_{\varepsilon}(t) \mathcal{B}^{0} \subset \mathcal{B}^{0}, \quad \forall t \geq 0 .
$$

Then, we state what is perhaps the main result of the paper.

Theorem 3.7. For every $\varepsilon \in\left[0, \varepsilon_{0}\right]$, there exists a set $\mathcal{K}_{\varepsilon}$, compact in $H^{1}$ and bounded in $H^{2}$, satisfying the following properties:

(i) The fractal dimension of $\mathcal{K}_{\varepsilon}$ in $H^{1}$ is uniformly bounded with respect to $\varepsilon$.

(ii) The set $\mathcal{K}_{\varepsilon}$ attracts any bounded subset $\mathcal{B} \subset H^{1}$ with an exponential rate which is uniform with respect to $\varepsilon$; namely, there exists $\kappa>0$ (independent of $\varepsilon$ and of the choice of $\mathcal{B}$ ) such that

$$
\boldsymbol{\delta}_{H^{1}}\left(U_{\varepsilon}(t) \mathcal{B}, \mathcal{K}_{\varepsilon}\right) \leq \mathcal{Q}\left(\|\mathcal{B}\|_{H^{1}}\right) e^{-\kappa t} .
$$

(iii) There exists $\tau>0$ such that

$$
\delta_{H^{1}}^{\mathrm{sym}}\left(\mathcal{K}_{\varepsilon}, \mathcal{K}_{0}\right) \leq C \varepsilon^{\tau} .
$$

The last theorems deal with the asymptotic behavior of single trajectories. We denote by $\mathcal{S}$ the set of stationary points or equilibria of (1.1), that is, the solutions to the elliptic problem

$$
\left\{\begin{array}{l}
-\Delta u^{\star}+\phi\left(u^{\star}\right)=0, \\
u_{\mid \partial \Omega}^{\star}=0 .
\end{array}\right.
$$

Theorem 3.8. For every $\varepsilon \in\left[0, \varepsilon_{0}\right]$ and $u_{0} \in H^{1}$, we have

$$
\lim _{t \rightarrow \infty}\left[\inf _{u^{\star} \in \mathcal{S}}\left\|\nabla U_{\varepsilon}(t) u_{0}-\nabla u^{\star}\right\|\right]=0
$$

and, if $\varepsilon>0$,

$$
\lim _{t \rightarrow \infty}\left\|\partial_{t} U_{\varepsilon}(t) u_{0}\right\|=0
$$


REMARK 3.9. In particular, if $\mathcal{S}$ is a discrete set, it follows that

$$
\lim _{t \rightarrow \infty}\left\|\nabla U_{\varepsilon}(t) u_{0}-\nabla u^{\star}\right\|=0,
$$

for some $u^{\star} \in \mathcal{S}$, depending on $\varepsilon \in\left[0, \varepsilon_{0}\right]$ and on the choice of $u_{0} \in H^{1}$.

Convergence of all trajectories to equilibria is, however, ensured, provided that the nonlinearity $\phi$ is real analytic.

Theorem 3.10. Let $\phi$ be real analytic. Then, for every fixed $\varepsilon \in\left[0, \varepsilon_{0}\right]$ and $u_{0} \in H^{1}$, there exists $u^{\star} \in \mathcal{S}$ such that

$$
\left\|\nabla U_{\varepsilon}(t) u_{0}-\nabla u^{\star}\right\| \leq \frac{c}{t^{\theta}}
$$

for some $\theta=\theta\left(u^{\star}\right)>0$ and $c=c\left(\varepsilon, u_{0}\right) \geq 0$. If $\varepsilon>0$, we also have

$$
\left\|\partial_{t} U_{\varepsilon}(t) u_{0}\right\| \leq \frac{c}{t^{\theta}} \text {. }
$$

The remainder of the paper is devoted to the proofs of the stated results.

4. The continuous dependence estimate. We begin to show that the solutions to (1.4) - (1.5), if they exist, fulfill the continuous dependence estimate provided by Proposition 3.4. We need a preliminary lemma.

Lemma 4.1. For $\varepsilon \in(0,1]$ and $u \in L^{\infty}\left([0, T], H^{1}\right)$, set

$$
\mathcal{J}(t, s)=\left\|\int_{0}^{s} \nabla u(t-y) d y\right\|^{2}
$$

and define the positive functionals

$$
\Psi_{0}(u(t))=k_{\varepsilon}(t) \mathcal{J}(t, t)-\int_{0}^{t} k_{\varepsilon}^{\prime}(s) \mathcal{J}(t, s) d s
$$

and

$$
\Psi_{1}(u(t))=-\frac{1}{2} k_{\varepsilon}^{\prime}(t) \mathcal{J}(t, t)+\frac{1}{2} \int_{0}^{t} k_{\varepsilon}^{\prime \prime}(s) \mathcal{J}(t, s) d s .
$$

Then, for every $t \in[0, T]$, the following equality holds:

$$
-\int_{0}^{t} k_{\varepsilon}(s)\langle\Delta u(t-s), u(t)\rangle d s=\frac{1}{2} \frac{d}{d t} \Psi_{0}(u(t))+\Psi_{1}(u(t)) .
$$

Proof. By direct computation, using the properties of $k_{\varepsilon}(s)$ and integration by parts. Note that all the terms are well defined, thanks to the regularity of $u$.

Proposition 4.2. Let $\varepsilon \in[0,1]$ be fixed, and let $u^{1}, u^{2} \in L^{\infty}\left([0, T], H^{1}\right)$ be two solutions to (1.4) on $[0, T]$ with initial data $u_{1}$ and $u_{2}$, respectively. Then, for every $t \in[0, T]$,

$$
\left\|u^{1}(t)-u^{2}(t)\right\| \leq \mathcal{Q}(T)\left\|u_{1}-u_{2}\right\| .
$$

Proof. Fix $\varepsilon \in(0,1]$ (the case $\varepsilon=0$ is well known), and denote $\bar{u}(t)=u^{1}(t)-u^{2}(t)$. Taking the difference in (1.4), we are led to

$$
\partial_{t} \bar{u}-\int_{0}^{t} k_{\varepsilon}(s) \Delta \bar{u}(t-s) d s+\phi\left(u^{1}\right)-\phi\left(u^{2}\right)=0 .
$$


Multiplying the equation by $\bar{u}$, and using the preceding lemma, we obtain the equality

$$
\frac{1}{2} \frac{d}{d t}\left[\|\bar{u}\|^{2}+\Psi_{0}(\bar{u})\right]+\Psi_{1}(\bar{u})+\left\langle\phi\left(u^{1}\right)-\phi\left(u^{2}\right), u\right\rangle=0 .
$$

Due to (2.3),

$$
\left\langle\phi\left(u^{1}\right)-\phi\left(u^{2}\right), \bar{u}\right\rangle \geq-\ell\|\bar{u}\|^{2} .
$$

Thus, using the fact that $\Psi_{0}(\bar{u})$ and $\Psi_{1}(\bar{u})$ are positive, we end up with the differential inequality

$$
\frac{d}{d t}\left[\|\bar{u}\|^{2}+\Psi_{0}(\bar{u})\right] \leq 2 \ell\left[\|\bar{u}\|^{2}+\Psi_{0}(\bar{u})\right] .
$$

Since $\Psi_{0}(\bar{u}(0))=0$, the conclusion follows from the Gronwall lemma.

5. An equivalent problem. In order to proceed with the investigation, it is convenient to work with a reformulated version of the original problem (1.4)-(1.5). To this end, we differentiate (1.4) with respect to time, thus obtaining

$$
\partial_{t t} u-k_{\varepsilon}(0) \Delta u-\int_{0}^{t} k_{\varepsilon}^{\prime}(s) \Delta u(t-s) d s+\phi^{\prime}(u) \partial_{t} u=0 .
$$

Choosing now

$$
\beta \in(0, \alpha),
$$

we multiply the equation above times $\varepsilon$, and we add the result to (1.4) times $\beta$. This procedure leads to the following integro-differential wave equation with nonlinear damping:

$$
\varepsilon \partial_{t t} u-k(0) \Delta u+\sigma_{\varepsilon}(u) \partial_{t} u+\int_{0}^{t} \mu_{\varepsilon}(s) \Delta u(t-s) d s+\beta \phi(u)=0,
$$

supplemented with Dirichlet boundary conditions, where we defined

$$
\mu_{\varepsilon}(s)=-\varepsilon k_{\varepsilon}^{\prime}(s)-\beta k_{\varepsilon}(s)
$$

and

$$
\sigma_{\varepsilon}(u)=\beta+\varepsilon \phi^{\prime}(u)
$$

Properties of $\boldsymbol{\mu}_{\varepsilon}$. Calling

$$
\mu(s)=-k^{\prime}(s)-\beta k(s),
$$

it turns out that

$$
\mu_{\varepsilon}(s)=\frac{1}{\varepsilon} \mu\left(\frac{s}{\varepsilon}\right) .
$$

The function $\mu$ is summable, absolutely continuous and nonincreasing on $\mathbb{R}^{+}$(hence, nonnegative and vanishing at infinity). Indeed, in light of (1.6), and since $\beta<\alpha$,

$$
\mu^{\prime}(s)=-k^{\prime \prime}(s)-\beta k^{\prime}(s) \leq-k^{\prime \prime}(s)-\alpha k^{\prime}(s) \leq 0 .
$$

Furthermore, setting

$$
\delta=\alpha-\beta>0,
$$

the differential inequality

$$
\mu^{\prime}(s)+\delta \mu(s)=-k^{\prime \prime}(s)-\alpha k^{\prime}(s)-\delta \beta k(s) \leq-k^{\prime \prime}(s)-\alpha k^{\prime}(s) \leq 0
$$


holds for almost every $s>0$. Thus, for every $\varepsilon \in(0,1]$ and almost every $s>0$,

$$
\mu_{\varepsilon}^{\prime}(s)+\frac{\delta}{\varepsilon} \mu_{\varepsilon}(s) \leq 0 \text {. }
$$

As a consequence, if $s \geq s_{0}>0$,

$$
\mu_{\varepsilon}(s) \leq \mu_{\varepsilon}\left(s_{0}\right) e^{-\frac{\delta}{\varepsilon}\left(s-s_{0}\right)} .
$$

Properties of $\boldsymbol{\sigma}_{\varepsilon}$. On account of (2.3) and (2.5), we have that

$$
\sigma_{\varepsilon}(u) \leq C\left(1+|u|^{\frac{1}{\gamma}}\right)
$$

and

$$
\sigma_{\varepsilon}(u) \geq \beta-\varepsilon \ell \geq-\varepsilon \ell .
$$

Hence, setting $\varepsilon_{0} \in(0,1]$ such that

$$
\sigma_{0}=\beta-\varepsilon_{0} \ell>0,
$$

we obtain the control

$$
\sigma_{\varepsilon}(u) \geq \sigma_{0}, \quad \forall \varepsilon \in\left(0, \varepsilon_{0}\right] .
$$

Finally, by (2.4), up to possibly enlarging $C$,

$$
\left|\sigma_{\varepsilon}^{\prime}(u)\right| \leq C\left[\sigma_{\varepsilon}(u)\right]^{1-\gamma}, \quad \forall \varepsilon \in\left(0, \varepsilon_{0}\right] .
$$

REMARK 5.1. In fact, since our scheme works for all $\beta<\alpha$, any $\varepsilon_{0} \in(0,1]$ such that $\alpha-\varepsilon_{0} \ell>0$ will do.

Definition 5.2. A couple $\left(u, \partial_{t} u\right)$ with

$$
u \in L^{\infty}\left([0, T], H^{1}\right), \quad \partial_{t} u \in L^{\infty}\left([0, T], H^{0}\right)
$$

is a solution to (5.1) on the time interval $[0, T]$ with initial data $\left(u_{0}, v_{0}\right) \in H^{1} \times H^{0}$ if $u(0)=u_{0}, \partial_{t} u(0)=v_{0}$ and, for every $t \in(0, T]$ and every test function $w \in H^{1}$,

$\varepsilon\left\langle\partial_{t t} u, w\right\rangle-k(0)\langle\nabla u, \nabla w\rangle+\left\langle\sigma_{\varepsilon}(u) \partial_{t} u, w\right\rangle-\int_{0}^{t} \mu_{\varepsilon}(s)\langle\nabla u(t-s), \nabla w\rangle d s+\beta\langle\phi(u), w\rangle=0$.

Again, due to the control $\partial_{t t} u \in L^{\infty}\left([0, T], H^{-1}\right)$, which is read from the equation, together with the standard embeddings, the initial conditions make sense.

The next step is to establish a relationship between the two formulations.

Proposition 5.3. Let $\left(u, \partial_{t} u\right) \in L^{\infty}\left([0, T], H^{1}\right) \times L^{\infty}\left([0, T], H^{0}\right)$ be a solution to (5.1) on $[0, T]$ with $u(0)=u_{0} \in H^{1}$ and $\partial_{t} u(0)=-\phi\left(u_{0}\right) \in H^{0}$. Then, $u$ is a solution to (1.4) on $[0, T]$ with $u(0)=u_{0}$.

REMARK 5.4. On account of this result and Proposition 4.2, Proposition 3.2 is proved once we exhibit the existence of a solution to (5.1) with initial data $\left(u_{0},-\phi\left(u_{0}\right)\right)$. As a byproduct, such a solution to (5.1) is necessarily unique.

Proof. Let $\left(u, \partial_{t} u\right)$ be a solution to (5.1) on $[0, T]$ with initial data $\left(u_{0},-\phi\left(u_{0}\right)\right)$. Given any $w \in H^{1}$, let us define

$$
\mathcal{F}(t)=\left\langle\partial_{t} u(t), w\right\rangle+\int_{0}^{t} k_{\varepsilon}(s)\langle\nabla u(t-s), \nabla w\rangle d s+\langle\phi(u(t)), w\rangle .
$$


Then, $u$ is a solution to (1.4) with initial datum $u_{0}$ if and only if $\mathcal{F}(t)=0$ for all $t \in[0, T]$ and every $w \in H^{1}$. On the other hand, since $u$ solves (5.1), we have that

$$
\varepsilon \frac{d}{d t} \mathcal{F}(t)+\beta \mathcal{F}(t)=0
$$

for all $t \in[0, T]$ and every $w \in H^{1}$. Therefore,

$$
\mathcal{F}(t)=\mathcal{F}(0) e^{-\frac{\beta}{\varepsilon} t} .
$$

Since

$$
\mathcal{F}(0)=\left\langle\partial_{t} u(0), w\right\rangle+\left\langle\phi\left(u_{0}\right), w\right\rangle=0,
$$

we conclude that $\mathcal{F}(t)=0$.

In fact, in the subsequent section, we will provide an existence and uniqueness result for a system of differential equations of which (5.1) is a particular case.

6. A dynamical system in the history space framework. For $\varepsilon \in(0,1]$ and $r \in \mathbb{R}$, we introduce the $L^{2}$-weighted space

$$
\mathcal{M}_{\varepsilon}^{r}=L_{\mu_{\varepsilon}}^{2}\left(\mathbb{R}^{+}, H^{r+1}\right),
$$

endowed with the inner product

$$
\left\langle\eta_{1}, \eta_{2}\right\rangle_{\mathcal{M}_{\varepsilon}^{r}}=\int_{0}^{\infty} \mu_{\varepsilon}(s)\left\langle\eta_{1}(s), \eta_{2}(s)\right\rangle_{H^{r+1}} d s,
$$

and we denote by

$$
T_{\varepsilon}: \operatorname{dom}\left(T_{\varepsilon}\right) \subset \mathcal{M}_{\varepsilon}^{0} \rightarrow \mathcal{M}_{\varepsilon}^{0}
$$

the infinitesimal generator of the strongly continuous semigroup of right translations on $\mathcal{M}_{\varepsilon}^{0}$, namely

$$
T_{\varepsilon} \eta=-D \eta, \quad \operatorname{dom}\left(T_{\varepsilon}\right)=\left\{\eta \in \mathcal{M}_{\varepsilon}^{0}: D \eta \in \mathcal{M}_{\varepsilon}^{0}, \eta(0)=0\right\},
$$

where $D$ denotes the distributional derivative, and the equality $\eta(0)=0$ is understood as $\lim _{s \rightarrow 0}\|\eta(s)\|_{\mathcal{M}_{\varepsilon}^{0}}=0$. If $\eta \in \operatorname{dom}\left(T_{\varepsilon}\right)$, on account of (5.2), we have the relation (see [20])

$$
\left\langle T_{\varepsilon} \eta, \eta\right\rangle_{\mathcal{M}_{\varepsilon}^{0}}=\frac{1}{2} \int_{0}^{\infty} \mu_{\varepsilon}^{\prime}(s)\|\nabla \eta(s)\|^{2} d s \leq-\frac{\delta}{2 \varepsilon}\|\eta\|_{\mathcal{M}_{\varepsilon}^{0}}^{2} .
$$

Given $T>0$ and

$$
u \in L^{\infty}\left([0, T], H^{1}\right) \cap W^{1, \infty}\left([0, T], H^{0}\right),
$$

the Cauchy problem

$$
\left\{\begin{array}{l}
\partial_{t} \eta^{t}=T_{\varepsilon} \eta^{t}+\partial_{t} u(t), \quad t>0, \\
\eta^{0}(s)=\eta_{0}(s) \in \mathcal{M}_{\varepsilon}^{0}
\end{array}\right.
$$

in the unknown variable $\eta=\eta^{t}(\boldsymbol{x}, s): \Omega \times \mathbb{R}^{+} \times[0, T] \rightarrow \mathbb{R}$, has a unique mild solution $\eta \in C\left([0, T], \mathcal{M}_{\varepsilon}^{0}\right)$ in the sense of [39], which has the explicit representation formula 20, 38

$$
\eta^{t}(s)= \begin{cases}u(t)-u(t-s), & 0<s \leq t, \\ \eta_{0}(s-t)+u(t)-u(0), & s>t\end{cases}
$$

Observe that the variable $\eta$, first introduced by Dafermos [7] to deal with problems with memory, is ruled by the past history of $u$. 
At this point, for every $\varepsilon \in(0,1]$, we define the product Hilbert space

$$
\mathcal{H}_{\varepsilon}^{r}=H^{r+1} \times H^{r} \times \mathcal{M}_{\varepsilon}^{r}
$$

normed by

$$
\|(u, v, \eta)\|_{\mathcal{H}_{\varepsilon}^{r}}^{2}=\beta\|u\|_{H^{r+1}}^{2}+\varepsilon\|v\|_{H^{r}}^{2}+\|\eta\|_{\mathcal{M}_{\varepsilon}^{r}}^{2} .
$$

It is convenient to extend the definition for $\varepsilon=0$ as

$$
\mathcal{H}_{0}^{r}=H^{r+1} \times\{0\} \times\{0\} .
$$

Given $\varepsilon \in(0,1]$ and $\left(u_{0}, v_{0}, \eta_{0}\right) \in \mathcal{H}_{\varepsilon}^{0}$, we consider, for $t>0$, the system in the so-called history space framework

$$
\left\{\begin{array}{l}
\varepsilon \partial_{t t} u+\sigma_{\varepsilon}(u) \partial_{t} u-\beta \Delta u-\int_{0}^{\infty} \mu_{\varepsilon}(s) \Delta \eta(s) d s+\beta \phi(u)=0, \\
\partial_{t} \eta=T_{\varepsilon} \eta+\partial_{t} u
\end{array}\right.
$$

satisfying the initial conditions

$$
\left(u(0), \partial_{t} u(0), \eta^{0}\right)=\left(u_{0}, v_{0}, \eta_{0}\right) .
$$

Definition 6.1. A triplet $\left(u, \partial_{t} u, \eta\right)$ with

$$
u \in L^{\infty}\left([0, T], H^{1}\right), \quad \partial_{t} u \in L^{\infty}\left([0, T], H^{0}\right), \quad \eta \in C\left([0, T], \mathcal{M}_{\varepsilon}^{0}\right)
$$

is a solution to (6.3) on the time interval $[0, T]$ with initial data $\left(u_{0}, v_{0}, \eta_{0}\right) \in \mathcal{H}_{\varepsilon}^{0}$ if $u(0)=u_{0}, \partial_{t} u(0)=v_{0}, \eta^{0}=\eta_{0}$, and, for every $t \in(0, T], u$ solves the first equation in the variational sense, and $\eta$ is given by (6.2).

We now dwell on the particular instance of (6.3) with initial data of the form $\left(u_{0}, v_{0}, u_{0}\right)$. In that case, (6.2) reads

$$
\eta^{t}(s)= \begin{cases}u(t)-u(t-s), & 0<s \leq t \\ u(t), & s>t\end{cases}
$$

Accordingly,

$$
-\int_{0}^{\infty} \mu_{\varepsilon}(s) \Delta \eta^{t}(s) d s=-[k(0)-\beta] \Delta u(t)+\int_{0}^{t} \mu_{\varepsilon}(s) \Delta u(t-s) d s .
$$

We summarize this discussion into the following corollary.

Corollary 6.2. Let $\left(u, \partial_{t} u, \eta\right)$ be a solution to (6.3) on $[0, T]$ with initial data $\left(u_{0}, v_{0}, u_{0}\right)$. Then $\left(u, \partial_{t} u\right)$ is a solution to (5.1) on $[0, T]$ with initial data $\left(u_{0}, v_{0}\right)$.

Problem (6.3) without memory, namely, the wave equation with nonlinear damping, was studied in 36 (see also 37 for the three-dimensional case), under suitable assumptions on the damping term. The existence and uniqueness of a solution to (6.3) for all $\varepsilon \in(0,1]$ is established in the following theorem.

Theorem 6.3. For every $\varepsilon \in(0,1]$ and every $T>0$, (6.3) has a unique solution $z(t)=$ $\left(u(t), \partial_{t} u(t), \eta^{t}\right)$ on $[0, T]$ with initial data $\left(u_{0}, v_{0}, \eta_{0}\right) \in \mathcal{H}_{\varepsilon}^{0}$. Moreover, calling $z^{i}=$ $\left(u^{i}, \partial_{t} u^{i}, \eta^{i}\right)$, with $i=1,2$, two solutions corresponding to the initial data $z_{i}=\left(u_{i}, v_{i}, \eta_{i}\right)$ with $\left\|z_{i}\right\|_{\mathcal{H}_{\varepsilon}^{0}} \leq R$, the continuous dependence estimate

$$
\left\|z^{1}(t)-z^{2}(t)\right\|_{\mathcal{H}_{\varepsilon}^{-1}} \leq \mathcal{Q}\left(T+R+\frac{1}{\varepsilon}\right)\left\|z_{1}-z_{2}\right\|_{\mathcal{H}_{\varepsilon}^{0}}
$$


holds for every $t \in[0, T]$.

Proof. Throughout this proof, the generic constant $C$ may depend (increasingly) on $T$ and $R$. The existence of a solution on $[0, T]$ is established by means of a Galerkin approximation scheme (cf. [17, 38]), leaning on the energy estimate

$$
\|z(t)\|_{\mathcal{H}_{\varepsilon}^{0}} \leq C, \quad \forall t \in[0, T]
$$

given by the subsequent Remark 7.3. Perhaps, the only nontrivial point in the passage to the limit is to identify the term $\left\langle\sigma_{\varepsilon}(u) \partial_{t} u, w\right\rangle$. Indeed, we have an approximating sequence $u^{n}$ that, up to a subsequence, converges to $u$ almost everywhere in $\Omega \times[0, T]$. Besides, $u^{n}$ is bounded in every $L^{p}(\Omega \times[0, T])$. Introducing

$$
\Sigma(u)=\int_{0}^{u} \sigma_{\varepsilon}(y) d y,
$$

and choosing any function $q \in C_{\mathrm{cpt}}^{\infty}([0, T])$, we have (as $\left.n \rightarrow \infty\right)$

$$
\begin{aligned}
\int_{0}^{T}\left\langle\sigma_{\varepsilon}\left(u^{n}(t)\right) \partial_{t} u^{n}(t), q(t) w\right\rangle d t & =\int_{0}^{T}\left\langle\partial_{t} \Sigma\left(u^{n}(t)\right), q(t) w\right\rangle d t \\
& =-\int_{0}^{T}\left\langle\Sigma\left(u^{n}(t)\right), q^{\prime}(t) w\right\rangle d t \\
& \rightarrow-\int_{0}^{T}\left\langle\Sigma(u(t)), q^{\prime}(t) w\right\rangle d t \\
& =\int_{0}^{T}\left\langle\sigma_{\varepsilon}(u(t)) \partial_{t} u(t), q(t) w\right\rangle d t
\end{aligned}
$$

since $\Sigma\left(u^{n}\right) \rightarrow \Sigma(u)$ almost everywhere in $\Omega \times[0, T]$ and, thanks to (5.4), $\Sigma\left(u^{n}\right)$ is uniformly bounded (with respect to $n$ ) in $L^{1+\frac{1}{\gamma}}(\Omega \times[0, T]$ ).

The proof of the continuous dependence closely follows the analogous ones presented in [36, 37. For the reader's convenience, we report it in some detail. Denote

$$
\bar{z}(t)=\left(\bar{u}(t), \partial_{t} \bar{u}(t), \bar{\eta}^{t}\right)=z^{1}(t)-z^{2}(t) .
$$

Integrating (6.3) on $(0, t)$, the functions

$$
w(t)=\int_{0}^{t} \bar{u}(\tau) d \tau \quad \text { and } \quad \psi^{t}(s)=\int_{0}^{t} \bar{\eta}^{\tau}(s) d \tau
$$

solve the system

$$
\left\{\begin{array}{l}
\varepsilon \partial_{t t} w+\Sigma\left(u^{1}\right)-\Sigma\left(u^{2}\right)-\beta \Delta w-\int_{0}^{\infty} \mu_{\varepsilon}(s) \Delta \psi(s) d s=F+G \\
\partial_{t} \psi=T_{\varepsilon} \psi+\partial_{t} w+\bar{\eta}^{0}-\bar{u}(0)
\end{array}\right.
$$

having defined

$$
F(t)=-\beta \int_{0}^{t}\left[\phi\left(u^{1}(\tau)\right)-\phi\left(u^{2}(\tau)\right)\right] d \tau, \quad G=\Sigma\left(u_{1}\right)-\Sigma\left(u_{2}\right)+\varepsilon \partial_{t} \bar{u}(0) .
$$


Multiplying the first equation of (6.5) by $\partial_{t} w=\bar{u}$ and the second by $\psi$ in $\mathcal{M}_{\varepsilon}^{0}$, and using (6.1), we obtain

$$
\begin{aligned}
\frac{1}{2} \frac{d}{d t}\|\zeta\|_{\mathcal{H}_{\varepsilon}^{0}}^{2}+\frac{\delta}{2 \varepsilon}\|\psi\|_{\mathcal{M}_{\varepsilon}^{0}}^{2} \leq & -\left\langle\Sigma\left(u^{1}\right)-\Sigma\left(u^{2}\right), \bar{u}\right\rangle+\left\langle\bar{\eta}^{0}-\bar{u}(0), \psi\right\rangle_{\mathcal{M}_{\varepsilon}^{0}} \\
& +\frac{d}{d t}\langle F, w\rangle+\frac{d}{d t}\langle G, w\rangle-\left\langle\partial_{t} F, w\right\rangle,
\end{aligned}
$$

where we set

$$
\zeta(t)=\left(w(t), \partial_{t} w(t), \psi^{t}\right)
$$

In light of (5.5),

$$
-\left\langle\Sigma\left(u^{1}\right)-\Sigma\left(u^{2}\right), \bar{u}\right\rangle \leq \varepsilon \ell\|\bar{u}\|^{2} .
$$

Besides,

$$
\left\langle\bar{\eta}^{0}-\bar{u}(0), \psi\right\rangle_{\mathcal{M}_{\varepsilon}^{0}} \leq \frac{\delta}{2 \varepsilon}\|\psi\|_{\mathcal{M}_{\varepsilon}^{0}}^{2}+C\|\bar{z}(0)\|_{\mathcal{H}_{\varepsilon}^{0}}^{2} .
$$

Thus, an integration in time over $(0, t)$, with $t \leq T$, leads to

$$
\begin{aligned}
\frac{1}{2}\|\zeta(t)\|_{\mathcal{H}_{\varepsilon}^{0}}^{2} \leq & C\|\bar{z}(0)\|_{\mathcal{H}_{\varepsilon}^{0}}^{2}+\varepsilon \ell \int_{0}^{t}\|\bar{u}(\tau)\|^{2} d \tau+\langle F(t), w(t)\rangle \\
& +\langle G, w(t)\rangle-\int_{0}^{t}\left\langle\partial_{t} F(y), w(y)\right\rangle d y \\
\leq & \frac{1}{4}\|\zeta(t)\|_{\mathcal{H}_{\varepsilon}^{0}}^{2}+C\|\bar{z}(0)\|_{\mathcal{H}_{\varepsilon}^{0}}^{2}+C \int_{0}^{t}\|\zeta(\tau)\|_{\mathcal{H}_{\varepsilon}^{0}}^{2} d \tau \\
& +C\left(\|F(t)\|_{H^{-1}}^{2}+\|G\|_{H^{-1}}^{2}+\int_{0}^{t}\left\|\partial_{t} F(\tau)\right\|_{H^{-1}}^{2} d \tau\right) .
\end{aligned}
$$

On account of (2.5), (5.4), and (6.4), we have

$$
\|F(t)\|_{H^{-1}}^{2}+\|G\|_{H^{-1}}^{2}+\int_{0}^{t}\left\|\partial_{t} F(\tau)\right\|_{H^{-1}}^{2} d \tau \leq C\|\bar{z}(0)\|_{\mathcal{H}_{\varepsilon}^{0}}^{2}+\frac{C}{\varepsilon} \int_{0}^{t}\|\zeta(\tau)\|_{\mathcal{H}_{\varepsilon}^{0}}^{2} d \tau .
$$

Hence, we end up with the inequality

$$
\|\zeta(t)\|_{\mathcal{H}_{\varepsilon}^{0}}^{2} \leq C\|\bar{z}(0)\|_{\mathcal{H}_{\varepsilon}^{0}}^{2}+\frac{C}{\varepsilon} \int_{0}^{t}\|\zeta(\tau)\|_{\mathcal{H}_{\varepsilon}^{0}}^{2} d \tau
$$

and the Gronwall lemma yields

$$
\|\bar{u}(t)\|^{2} \leq\|\zeta(t)\|_{\mathcal{H}_{\varepsilon}^{0}}^{2} \leq C e^{\frac{C}{\varepsilon}}\|\bar{z}(0)\|_{\mathcal{H}_{\varepsilon}^{0}}^{2}
$$

Then, the analogous control on $\varepsilon\left\|\partial_{t} \bar{u}\right\|_{H^{-1}}=\varepsilon\left\|\partial_{t t} w\right\|_{H^{-1}}$ is obtained by comparison in the first equation of (6.5). Finally, using the representation formula (6.2) for $\bar{\eta}$, we easily recover the remaining estimate for $\|\bar{\eta}\|_{\mathcal{M}_{\varepsilon}^{-1}}$.

In conclusion, for every $\varepsilon \in(0,1]$, system (6.3) generates a semigroup

$$
S_{\varepsilon}(t): \mathcal{H}_{\varepsilon}^{0} \rightarrow \mathcal{H}_{\varepsilon}^{0}
$$

defined by

$$
S_{\varepsilon}(t)\left(u_{0}, v_{0}, \eta_{0}\right)=\left(u(t), \partial_{t} u(t), \eta^{t}\right) .
$$

We extend the definition to the case $\varepsilon=0$ by introducing $S_{0}(t): \mathcal{H}_{0}^{0} \rightarrow \mathcal{H}_{0}^{0}$ as

$$
S_{0}(t)\left(u_{0}, 0,0\right)=\left(U_{0}(t) u_{0}, 0,0\right) .
$$


In the next sections, we will analyze the asymptotic properties of the family of semigroups $S_{\varepsilon}(t)$.

7. Dissipativity. Our first result details the dissipative character of $S_{\varepsilon}(t)$. The value $\varepsilon_{0}$ appearing here and in the sequel is given by (5.6).

Proposition 7.1. For every $\varepsilon \in\left[0, \varepsilon_{0}\right]$, the semigroup $S_{\varepsilon}(t)$ possesses an absorbing set $\mathbb{B}_{\varepsilon}^{0} \subset \mathcal{H}_{\varepsilon}^{0}$, which is bounded in $\mathcal{H}_{\varepsilon}^{0}$ with a bound independent of $\varepsilon$. Precisely, for any bounded set $\mathbb{B} \subset \mathcal{H}_{\varepsilon}^{0}$, there exists a time $t_{0} \geq 0$, depending on the norm of $\mathbb{B}$ but independent of $\varepsilon$, such that

$$
S_{\varepsilon}(t) \mathbb{B} \subset \mathbb{B}_{\varepsilon}^{0}, \quad \forall t \geq t_{0} .
$$

The result is a direct consequence of the following lemma.

Lemma 7.2. There exists $C_{0} \geq 0$ such that, for any $\varepsilon \in\left[0, \varepsilon_{0}\right]$,

$$
\left\|S_{\varepsilon}(t) z\right\|_{\mathcal{H}_{\varepsilon}^{0}} \leq \mathcal{Q}\left(\|z\|_{\mathcal{H}_{\varepsilon}^{0}}\right) e^{-t}+C_{0}
$$

Moreover,

$$
\sigma_{0} \int_{0}^{\infty}\left\|\partial_{t} u(t)\right\|^{2} d t \leq \int_{0}^{\infty}\left\langle\sigma_{\varepsilon}(u(t)) \partial_{t} u(t), \partial_{t} u(t)\right\rangle d t \leq \mathcal{Q}\left(\|z\|_{\mathcal{H}_{\varepsilon}^{0}}\right) .
$$

Proof. We assume $\varepsilon>0$, since the proof of the case $\varepsilon=0$ is well known. Consider the energy functional

$$
L(t)=\left\|S_{\varepsilon}(t) z\right\|_{\mathcal{H}_{\varepsilon}^{0}}^{2}+2 \beta\langle\Phi(u(t)), 1\rangle
$$

with $\Phi(u)=\int_{0}^{u} \phi(y) d y$. In light of (2.2), there exists some $\vartheta>0$ such that

$$
\begin{gathered}
\|\nabla u\|^{2}+2\langle\Phi(u), 1\rangle \geq 2 \vartheta\|\nabla u\|^{2}-C, \\
\|\nabla u\|^{2}+\langle\phi(u), u\rangle \geq \vartheta\|\nabla u\|^{2}-C .
\end{gathered}
$$

Hence, using (2.5), and assuming without loss of generality that $\vartheta \leq 1 / 2$,

$$
2 \vartheta\left\|S_{\varepsilon}(t) z\right\|_{\mathcal{H}_{\varepsilon}^{0}}^{2}-C \leq L(t) \leq \mathcal{Q}\left(\left\|S_{\varepsilon}(t) z\right\|_{\mathcal{H}_{\varepsilon}^{0}}\right)+C .
$$

Multiplying the first equation of (6.3) by $\partial_{t} u$ in $H^{0}$ and the second by $\eta$ in $\mathcal{M}_{\varepsilon}^{0}$, thanks to (6.1), we obtain

$$
\frac{d}{d t} L+2\left\langle\sigma_{\varepsilon}(u) \partial_{t} u, \partial_{t} u\right\rangle+\frac{\delta}{\varepsilon}\|\eta\|_{\mathcal{M}_{\varepsilon}^{0}}^{2} \leq 0
$$

Keeping in mind (5.7), an integration of (7.2) yields

$$
\left\|S_{\varepsilon}(t) z\right\|_{\mathcal{H}_{\varepsilon}^{0}} \leq \mathcal{Q}\left(\|z\|_{\mathcal{H}_{\varepsilon}^{0}}\right), \quad \forall t \geq 0
$$

together with the sought integral estimate (17.1). To complete the proof, for $\omega>0$ to be fixed later, we define

$$
\Lambda(t)=L(t)+2 \omega \varepsilon\left\langle u(t), \partial_{t} u(t)\right\rangle+2 \omega\langle\Upsilon(u(t)), 1\rangle,
$$

with $\Upsilon(u)=\int_{0}^{u} y \sigma_{\varepsilon}(y) d y$. Since $\langle\Upsilon(u), 1\rangle \geq 0$, it is easy to see that, for $\omega$ small enough,

$$
\vartheta\left\|S_{\varepsilon}(t) z\right\|_{\mathcal{H}_{\varepsilon}^{0}}^{2}-C \leq \Lambda(t) \leq \mathcal{Q}\left(\left\|S_{\varepsilon}(t) z\right\|_{\mathcal{H}_{\varepsilon}^{0}}\right)+C .
$$


Then, multiplying the first equation of (6.3) by $\omega u$ and adding the result to (7.2), we have

$$
\frac{d}{d t} \Lambda+2 \omega \vartheta \beta\|\nabla u\|^{2}+2\left(\sigma_{0}-\omega \varepsilon\right)\left\|\partial_{t} u\right\|^{2}+\frac{\delta}{\varepsilon}\|\eta\|_{\mathcal{M}_{\varepsilon}^{0}}^{2} \leq-2 \omega\langle\eta, u\rangle_{\mathcal{M}_{\varepsilon}^{0}}+C .
$$

Up to further reducing $\omega$, we find the controls

$$
\omega \vartheta \leq \delta / 2, \quad 2\left(\sigma_{0}-\omega \varepsilon\right) \geq \omega \vartheta \varepsilon
$$

and

$$
-2 \omega\langle\eta, u\rangle_{\mathcal{M}_{\varepsilon}^{0}} \leq \frac{\delta}{2 \varepsilon}\|\eta\|_{\mathcal{M}_{\varepsilon}^{0}}^{2}+\omega \vartheta \beta\|\nabla u\|^{2},
$$

which lead to the differential inequality

$$
\frac{d}{d t} \Lambda(t)+\omega \vartheta\left\|S_{\varepsilon}(t) z\right\|_{\mathcal{H}_{\varepsilon}^{0}}^{2} \leq C .
$$

A generalized version of the Gronwall lemma (see [3]) provides the existence of $C_{0} \geq 0$ and a time $t_{0}=t_{0}\left(\|z\|_{\mathcal{H}_{\varepsilon}^{0}}\right)>0$ such that

$$
\left\|S_{\varepsilon}(t) z\right\|_{\mathcal{H}_{\varepsilon}^{0}} \leq C_{0}, \quad \forall t \geq t_{0} .
$$

Combining this estimate with (7.3), the conclusion follows.

REMARK 7.3. In fact, an energy estimate on finite time intervals holds for all $\varepsilon \in[0,1]$, namely,

$$
\left\|S_{\varepsilon}(t) z\right\|_{\mathcal{H}_{\varepsilon}^{0}} \leq \mathcal{Q}(T+R), \quad \forall t \in[0, T],
$$

whenever $\|z\|_{\mathcal{H}_{\varepsilon}^{0}} \leq R$. This is obtained reasoning as in the first part of the proof above; the only difference is the role of the term $\left\langle\sigma_{\varepsilon}(u) \partial_{t} u, \partial_{t} u\right\rangle$ in (17.2), which, due to (2.3), is now controlled as

$$
-\left\langle\sigma_{\varepsilon}(u) \partial_{t} u, \partial_{t} u\right\rangle \leq \varepsilon \ell\left\|\partial_{t} u\right\|^{2} \leq \ell L+C .
$$

The Gronwall lemma completes the argument.

8. Higher-order dissipativity. A further step is to prove the existence of an absorbing set in a more regular phase space compactly embedded into $\mathcal{H}_{\varepsilon}^{0}$. We first note that, for $\varepsilon>0$, the embedding $\mathcal{H}_{\varepsilon}^{1} \subset \mathcal{H}_{\varepsilon}^{0}$ is not compact, due to the lack of compactness of $\mathcal{M}_{\varepsilon}^{1} \subset \mathcal{M}_{\varepsilon}^{0}$ (see [38]). Thus, following the lines of [6, 13], we introduce the Banach space

$$
\mathcal{L}_{\varepsilon}=\left\{\eta \in \mathcal{M}_{\varepsilon}^{1} \cap \operatorname{dom}\left(T_{\varepsilon}\right): \sup _{x \geq 1} x \mathbb{T}_{\eta}^{\varepsilon}(x)<\infty\right\},
$$

where $\mathbb{T}_{\eta}^{\varepsilon}$ is the tail function of $\eta$, given by

$$
\mathbb{T}_{\eta}^{\varepsilon}(x)=\int_{\left(0, \frac{\varepsilon}{x}\right) \cup(\varepsilon x, \infty)} \mu_{\varepsilon}(s)\|\nabla \eta(s)\|^{2} d s
$$

endowed with the norm

$$
\|\eta\|_{\mathcal{L}_{\varepsilon}}^{2}=\|\eta\|_{\mathcal{M}_{\varepsilon}^{1}}^{2}+\|\eta\|_{\varepsilon}^{2}
$$


having set 1

$$
\|\eta\|_{\varepsilon}^{2}=\varepsilon^{2}\left\|T_{\varepsilon} \eta\right\|_{\mathcal{M}_{\varepsilon}^{0}}^{2}+\sup _{x \geq 1} x \mathbb{T}_{\eta}^{\varepsilon}(x) .
$$

Finally, for $\varepsilon>0$, we consider the Banach space

$$
\mathcal{Z}_{\varepsilon}=H^{2} \times H^{1} \times \mathcal{L}_{\varepsilon},
$$

which, according to [6], is compactly embedded into $\mathcal{H}_{\varepsilon}^{0}$. Again, we extend the definition to $\varepsilon=0$ by setting $\mathcal{Z}_{0}=\mathcal{H}_{0}^{1}$. The following fact has been mentioned in [6, 13] without proof.

Proposition 8.1. Closed balls of $\mathcal{Z}_{\varepsilon}$ are compact in $\mathcal{H}_{\varepsilon}^{0}$.

Proof. We just have to prove that closed balls of $\mathcal{Z}_{\varepsilon}$ are closed in $\mathcal{H}_{\varepsilon}^{0}$. In fact, it is enough to prove that closed balls centered at zero of $\mathcal{L}_{\varepsilon}$ are closed in $\mathcal{M}_{\varepsilon}^{0}$. Let $\eta_{n} \in \mathcal{L}_{\varepsilon}$ be such that $\left\|\eta_{n}\right\|_{\mathcal{L}_{\varepsilon}}^{2} \leq R$ and $\eta_{n} \rightarrow \eta$ in $\mathcal{M}_{\varepsilon}^{0}$. Then, up to a subsequence, $\eta_{n} \rightarrow \eta$ weakly in the Hilbert space $\mathcal{M}_{\varepsilon}^{1} \cap H_{\mu_{\varepsilon}}^{1}\left(\mathbb{R}^{+}, H^{1}\right)$. Moreover, $\eta(0)=0$, which can be argued by using the fact that $\left\|\eta_{n}(s)\right\|_{H^{1}} \rightarrow 0$ uniformly as $s \rightarrow 0$ (cf. [20]). Hence,

$$
\|\eta\|_{\mathcal{M}_{\varepsilon}^{1}}^{2}+\varepsilon^{2}\left\|T_{\varepsilon} \eta\right\|_{\mathcal{M}_{\varepsilon}^{0}}^{2} \leq \liminf _{n \rightarrow \infty}\left[\left\|\eta_{n}\right\|_{\mathcal{M}_{\varepsilon}^{1}}^{2}+\varepsilon^{2}\left\|T_{\varepsilon} \eta_{n}\right\|_{\mathcal{M}_{\varepsilon}^{0}}^{2}\right]
$$

The convergence in $\mathcal{M}_{\varepsilon}^{0}$ implies that, for every $y \geq 1$,

$$
y \mathbb{T}_{\eta}^{\varepsilon}(y)=\liminf _{n \rightarrow \infty} y \mathbb{T}_{\eta_{n}}^{\varepsilon}(y) \leq \liminf _{n \rightarrow \infty}\left[\sup _{x \geq 1} x \mathbb{T}_{\eta_{n}}^{\varepsilon}(x)\right],
$$

and, taking the supremum,

$$
\sup _{x \geq 1} x \mathbb{T}_{\eta}^{\varepsilon}(x) \leq \liminf _{n \rightarrow \infty}\left[\sup _{x \geq 1} x \mathbb{T}_{\eta_{n}}^{\varepsilon}(x)\right] .
$$

Collecting the two estimates above, we conclude that

$$
\|\eta\|_{\mathcal{L}_{\varepsilon}}^{2} \leq \liminf _{n \rightarrow \infty}\left[\left\|\eta_{n}\right\|_{\mathcal{M}_{\varepsilon}^{1}}^{2}+\varepsilon^{2}\left\|T_{\varepsilon} \eta_{n}\right\|_{\mathcal{M}_{\varepsilon}^{0}}^{2}\right]+\liminf _{n \rightarrow \infty}\left[\sup _{x \geq 1} x \mathbb{T}_{\eta_{n}}^{\varepsilon}(x)\right] \leq \liminf _{n \rightarrow \infty}\left\|\eta_{n}\right\|_{\mathcal{L}_{\varepsilon}}^{2} \leq R
$$

which proves the assertion.

The higher-order dissipativity of $S_{\varepsilon}(t)$ reads as follows.

Proposition 8.2. For every $\varepsilon \in\left[0, \varepsilon_{0}\right]$, the semigroup $S_{\varepsilon}(t)$ maps $\mathcal{Z}_{\varepsilon}$ into $\mathcal{Z}_{\varepsilon}$, and the restriction of $S_{\varepsilon}(t)$ to $\mathcal{Z}_{\varepsilon}$ possesses a bounded absorbing set $\mathbb{B}_{\varepsilon}^{1} \subset \mathcal{Z}_{\varepsilon}$, with a bound independent of $\varepsilon$.

The proof of this proposition will be carried out through several lemmas. In the sequel, we will always assume $\varepsilon \leq \varepsilon_{0}$. We first report a generalized version of the Gronwall lemma.

${ }^{1}$ We take here the occasion to correct the definition of the higher norm of $\eta$ adopted in [6, 13, although the mistake was not influent in those papers. With this choice, the map $\eta(s) \mapsto \eta\left(\varepsilon s / \varepsilon_{0}\right)$ is an isometric isomorphism from $\mathcal{L}_{\varepsilon}$ into $\mathcal{L}_{\varepsilon_{0}}$, which is onto if $\varepsilon>0$. Thus, for every $r>0$ and every $\varepsilon \in\left[0, \varepsilon_{0}\right]$, the minimum number of $r$-balls of $\mathcal{H}_{\varepsilon}^{0}$ needed to cover the unit ball of $\mathcal{Z}_{\varepsilon}$ is equal to (or less than, if $\varepsilon=0$ ) the minimum number of $r$-balls of $\mathcal{H}_{\varepsilon_{0}}^{0}$ needed to cover the unit ball of $\mathcal{Z}_{\varepsilon_{0}}$ (see 6 , Theorem A.2]). 
Lemma 8.3. Let $\Lambda:[0, \infty) \rightarrow[0, \infty)$ be an absolutely continuous function satisfying

$$
\frac{d}{d t} \Lambda(t)+2 \nu \Lambda(t) \leq h(t) \Lambda(t)+k
$$

where $\nu>0, k \geq 0$ and $\int_{s}^{t} h(\tau) d \tau \leq \nu(t-s)+m$, for all $t \geq s \geq 0$ and some $m \geq 0$. Then,

$$
\Lambda(t) \leq \Lambda(0) e^{m} e^{-\nu t}+\frac{k e^{m}}{\nu} .
$$

Lemma 8.4. Let $p, q \geq 1$ be such that $\frac{1}{p}+\frac{1}{q}=2 \gamma$, with $\gamma$ as in (5.8), and let $a, b \in H^{1}$. Then

$$
\left\langle\left[\sigma_{\varepsilon}^{\prime}(u)\right]^{2}\left[\sigma_{\varepsilon}(u)\right]^{-1} a^{2}, b^{2}\right\rangle \leq C\left\langle\sigma_{\varepsilon}(u) a, a\right\rangle^{1-2 \gamma}\|a\|^{\frac{2}{q}}\|b\|^{\frac{2}{p}}\|\nabla a\|^{\frac{2}{p}}\|\nabla b\|^{2-\frac{2}{p}} .
$$

Proof. The Hölder inequality with exponents $\left(\frac{1}{1-2 \gamma}, p, q\right)$, along with (5.8), entail

$$
\begin{aligned}
\left\langle\left[\sigma_{\varepsilon}^{\prime}(u)\right]^{2}\left[\sigma_{\varepsilon}(u)\right]^{-1} a^{2}, b^{2}\right\rangle & =\left\langle\left[\sigma_{\varepsilon}^{\prime}(u)\right]^{2}\left[\sigma_{\varepsilon}(u)\right]^{-1} a^{2-4 \gamma}, b^{2} a^{4 \gamma}\right\rangle \\
& \leq C\left\langle\sigma_{\varepsilon}(u) a, a\right\rangle^{1-2 \gamma}\|b\|_{L^{2 p}}^{2}\|a\|_{L^{4 \gamma q}}^{4 \gamma} .
\end{aligned}
$$

Exploiting (2.1),

$$
\begin{aligned}
\|b\|_{L^{2 p}}^{2} & \leq C\|b\|^{\frac{2}{p}}\|\nabla b\|^{2-\frac{2}{p}} \\
\|a\|_{L^{4 \gamma q}}^{4 \gamma} & \leq C\|a\|^{\frac{2}{q}}\|\nabla a\|^{4 \gamma-\frac{2}{q}} .
\end{aligned}
$$

Collecting the estimates, and noting that $4 \gamma-\frac{2}{q}=\frac{2}{p}$, will do.

Lemma 8.5. There exists $\nu_{1}>0$ such that

$$
\left\|S_{\varepsilon}(t) z\right\|_{\mathcal{H}_{\varepsilon}^{1}} \leq \mathcal{Q}\left(\|z\|_{\mathcal{H}_{\varepsilon}^{1}}\right) e^{-\nu_{1} t}+\mathcal{Q}(R),
$$

whenever $z \in \mathcal{H}_{\varepsilon}^{1}$ with $\|z\|_{\mathcal{H}_{\varepsilon}^{0}} \leq R$.

Proof. Assume $\varepsilon>0$. In the proof, the generic constant $C$ may depend (increasingly) on $R$. Let $z \in \mathcal{H}_{\varepsilon}^{1}$ be such that $\|z\|_{\mathcal{H}_{\varepsilon}^{0}} \leq R$. In particular, from Lemma 7.2 we know that $\left\|S_{\varepsilon}(t) z\right\|_{\mathcal{H}_{\varepsilon}^{0}} \leq C$. For $\omega>0$ to be fixed later, we introduce the functional

$$
\Lambda(t)=\left\|S_{\varepsilon}(t) z\right\|_{\mathcal{H}_{\varepsilon}^{1}}^{2}-2 \beta\langle\phi(u), \Delta u\rangle-2 \varepsilon \omega\left\langle\partial_{t} u, \Delta u\right\rangle+C .
$$

Then, if $\omega$ is small enough and the constant $C$ above is large enough,

$$
\frac{1}{2}\left\|S_{\varepsilon}(t) z\right\|_{\mathcal{H}_{\varepsilon}^{1}}^{2} \leq \Lambda(t) \leq 2\left\|S_{\varepsilon}(t) z\right\|_{\mathcal{H}_{\varepsilon}^{1}}^{2}+C .
$$

Multiplying the first equation in (6.3) by $-\Delta \partial_{t} u-\omega \Delta u$ in $H^{0}$ and the second by $\eta$ in $\mathcal{M}_{\varepsilon}^{1}$, taking into account (5.7) and (6.1), and taking $\omega \leq \sigma_{0} / 2$, we obtain

$$
\begin{aligned}
& \frac{d \Lambda}{d t}+2 \omega \beta\|\Delta u\|^{2}+\frac{\sigma_{0}}{2}\left\|\nabla \partial_{t} u\right\|^{2}+\frac{\delta}{\varepsilon}\|\eta\|_{\mathcal{M}_{\varepsilon}^{1}}^{2} \\
& \leq-2 \beta\left\langle\phi^{\prime}(u) \partial_{t} u, \Delta u\right\rangle+2 \omega\left\langle\sigma_{\varepsilon}(u) \partial_{t} u, \Delta u\right\rangle-2 \omega\langle\eta, u\rangle_{\mathcal{M}_{\varepsilon}^{1}}+2 \omega \beta\langle\phi(u), \Delta u\rangle \\
& \quad-2\left\langle\sigma_{\varepsilon}^{\prime}(u) \partial_{t} u \nabla u, \nabla \partial_{t} u\right\rangle-\left\langle\sigma_{\varepsilon}(u) \nabla \partial_{t} u, \nabla \partial_{t} u\right\rangle .
\end{aligned}
$$


In light of (2.1),

$$
\begin{aligned}
-2 \beta\left\langle\phi^{\prime}(u) \partial_{t} u, \Delta u\right\rangle+2 \omega\left\langle\sigma_{\varepsilon}(u) \partial_{t} u, \Delta u\right\rangle & \leq C\left\|\partial_{t} u\right\|^{\frac{1}{2}}\left\|\nabla \partial_{t} u\right\|^{\frac{1}{2}}\|\Delta u\| \\
& \leq \frac{\sigma_{0}}{8}\left\|\nabla \partial_{t} u\right\|^{2}+C\left\|\partial_{t} u\right\|\|\Delta u\|^{2}+C \\
& \leq \frac{\sigma_{0}}{8}\left\|\nabla \partial_{t} u\right\|^{2}+\frac{\omega \beta}{2}\|\Delta u\|^{2}+C\left\|\partial_{t} u\right\|^{2} \Lambda+C .
\end{aligned}
$$

Moreover,

$$
2 \omega \beta\langle\phi(u), \Delta u\rangle \leq \frac{\omega \beta}{2}\|\Delta u\|^{2}+C,
$$

and, up to reducing $\omega$,

$$
-2 \omega\langle\eta, u\rangle_{\mathcal{M}_{\varepsilon}^{1}} \leq \frac{\delta}{2 \varepsilon}\|\eta\|_{\mathcal{M}_{\varepsilon}^{1}}^{2}+\frac{\omega \beta}{2}\|\Delta u\|^{2} .
$$

Finally, it is readily seen that

$$
-2\left\langle\sigma_{\varepsilon}^{\prime}(u) \partial_{t} u \nabla u, \nabla \partial_{t} u\right\rangle-\left\langle\sigma_{\varepsilon}(u) \nabla \partial_{t} u, \nabla \partial_{t} u\right\rangle \leq\left\langle\left[\sigma_{\varepsilon}^{\prime}(u)\right]^{2}\left[\sigma_{\varepsilon}(u)\right]^{-1}\left[\partial_{t} u\right]^{2},|\nabla u|^{2}\right\rangle .
$$

Hence, in light of Lemma 8.4 with $a=\partial_{t} u$ and $b=|\nabla u|$, we have

$$
\begin{aligned}
& \left\langle\left[\sigma_{\varepsilon}^{\prime}(u)\right]^{2}\left[\sigma_{\varepsilon}(u)\right]^{-1}\left[\partial_{t} u\right]^{2},|\nabla u|^{2}\right\rangle \\
& \leq C\left\langle\sigma_{\varepsilon}(u) \partial_{t} u, \partial_{t} u\right\rangle^{1-2 \gamma}\left\|\partial_{t} u\right\|^{\frac{2}{q}}\left\|\nabla \partial_{t} u\right\|^{\frac{2}{p}}\|\Delta u\|^{2-\frac{2}{p}} \\
& =\frac{C\left\|\partial_{t} u\right\|^{\frac{2}{q}}}{\left\langle\sigma_{\varepsilon}(u) \partial_{t} u, \partial_{t} u\right\rangle^{\frac{1}{q}}}\left(\left\langle\sigma_{\varepsilon}(u) \partial_{t} u, \partial_{t} u\right\rangle\|\Delta u\|^{2}\right)^{1-\frac{1}{p}}\left\|\nabla \partial_{t} u\right\|^{\frac{2}{p}} \\
& \leq \frac{\sigma_{0}}{8}\left\|\nabla \partial_{t} u\right\|^{2}+C\left\langle\sigma_{\varepsilon}(u) \partial_{t} u, \partial_{t} u\right\rangle \Lambda .
\end{aligned}
$$

These computations entail the inequality

$$
\frac{d \Lambda}{d t}+4 \nu_{1} \Lambda \leq C\left\langle\sigma_{\varepsilon}(u) \partial_{t} u, \partial_{t} u\right\rangle \Lambda+C,
$$

for some $\nu_{1}>0$. The integral estimate (7.1) allows us to apply Lemma 8.3, which yields the desired conclusion. The case $\varepsilon=0$ is treated in the same manner.

The subsequent corollary will be needed in the next sections.

Corollary 8.6. If $z \in \mathcal{H}_{\varepsilon}^{1}$ with $\|z\|_{\mathcal{H}_{\varepsilon}^{1}} \leq R$, we have the integral control

$$
\int_{0}^{t}\left\|\nabla \partial_{t} u(\tau)\right\|^{2} d \tau \leq \mathcal{Q}(R)(1+t)
$$

Proof. Set $\omega=0$ and integrate (8.1) on $(0, t)$.

Lemma 8.7. Let $\eta$ be given by (6.2). Assume that $\eta_{0} \in \operatorname{dom}\left(T_{\varepsilon}\right)$ and

$$
\varepsilon\left\|\nabla \partial_{t} u(t)\right\|^{2} \leq \Theta,
$$

for some $\Theta \geq 0$ and every $t \geq 0$. Then, $\eta^{t} \in \operatorname{dom}\left(T_{\varepsilon}\right)$ and

$$
\left\|\eta^{t}\right\|_{\varepsilon}^{2} \leq C e^{-2 \delta t}\left\|\eta_{0}\right\|_{\varepsilon}^{2}+C \Theta .
$$

Proof. Argue exactly as in [6, with minor variations.

We are now ready to conclude our discussion. 
Proof of Proposition 8.2 . Due to the existence of the absorbing set $\mathbb{B}_{\varepsilon}^{0}$ in $\mathcal{H}_{\varepsilon}^{0}$, it suffices to show that

$$
\left\|S_{\varepsilon}(t) z\right\|_{\mathcal{Z}_{\varepsilon}} \leq \mathcal{Q}\left(\|z\|_{\mathcal{Z}_{\varepsilon}}\right) e^{-\nu_{1} t}+\mathcal{Q}(R),
$$

whenever $z \in \mathcal{H}_{\varepsilon}^{1}$ with $\|z\|_{\mathcal{H}_{\varepsilon}^{0}} \leq R$. In light of Lemma 8.5, we only need to control the norm $\left\|\eta^{t}\right\|_{\varepsilon}$. On the other hand, after Lemma 8.5, we know that

$$
\varepsilon\left\|\nabla \partial_{t} u(t)\right\|^{2} \leq \mathcal{Q}\left(\|z\|_{\mathcal{Z}_{\varepsilon}}\right) e^{-2 \nu_{1} t}+\mathcal{Q}(R) .
$$

Therefore, assuming without loss of generality that $\nu_{1} \leq \delta$, the desired inequality follows from an application of Lemma 8.7.

9. Continuous dependence for smoother initial data. With more regular initial data, we can improve the continuous dependence estimate of Theorem 6.3. In view of further scopes, we will actually consider the more general system

$$
\left\{\begin{array}{l}
\varepsilon \partial_{t t} u+\sigma_{\varepsilon}(u) \partial_{t} u-\beta \Delta u-\int_{0}^{\infty} \mu_{\varepsilon}(s) \Delta \eta(s) d s+\beta \phi(u)=f, \\
\partial_{t} \eta=T_{\varepsilon} \eta+\partial_{t} u,
\end{array}\right.
$$

where $f \in L_{\text {loc }}^{2}\left([0, \infty), H^{0}\right)$, whose solutions can be described by means of a one-parameter family of operators $S_{\varepsilon}^{f}(t)$ on $\mathcal{H}_{\varepsilon}^{0}$ (just adapt the proof of Theorem 6.3).

Proposition 9.1. Let $\varepsilon \in\left[0, \varepsilon_{0}\right]$ and $f \in L_{\mathrm{loc}}^{2}\left([0, \infty), H^{0}\right)$, and let $z_{1} \in \mathcal{H}_{\varepsilon}^{0}$ and $z_{2} \in \mathcal{H}_{\varepsilon}^{1}$ be such that

$$
\left\|z_{1}\right\|_{\mathcal{H}_{\varepsilon}^{0}}+\left\|z_{2}\right\|_{\mathcal{H}_{\varepsilon}^{1}} \leq R
$$

Then, we have the estimate

$$
\left\|S_{\varepsilon}^{f}(t) z_{1}-S_{\varepsilon}(t) z_{2}\right\|_{\mathcal{H}_{\varepsilon}^{0}}^{2} \leq \mathcal{Q}(R) e^{\mathcal{Q}(R) t}\left[\left\|z_{1}-z_{2}\right\|_{\mathcal{H}_{\varepsilon}^{0}}^{2}+\int_{0}^{t}\|f(\tau)\|^{2} d \tau\right] .
$$

Proof. In this proof, the generic constant $C \geq 0$ may depend (increasingly) on $R$. Consider the differential system solved by

$$
\bar{z}(t)=\left(\bar{u}(t), \partial_{t} \bar{u}(t), \bar{\eta}^{t}\right)=S_{\varepsilon}^{f}(t) z_{1}-S_{\varepsilon}(t) z_{2},
$$

namely,

$$
\left\{\begin{array}{l}
\varepsilon \partial_{t t} \bar{u}+\sigma_{\varepsilon}\left(u^{1}\right) \partial_{t} \bar{u}-\beta \Delta \bar{u}-\int_{0}^{\infty} \mu_{\varepsilon}(s) \Delta \bar{\eta}(s) d s=W+f, \\
\partial_{t} \bar{\eta}=T_{\varepsilon} \bar{\eta}+\partial_{t} \bar{u}
\end{array}\right.
$$

having put

$$
W=-\beta\left[\phi\left(u^{1}\right)-\phi\left(u^{2}\right)\right]-\left[\sigma_{\varepsilon}\left(u^{1}\right)-\sigma_{\varepsilon}\left(u^{2}\right)\right] \partial_{t} u^{2},
$$

where $u^{1}(t)$ and $u^{2}(t)$ are the first components of $S_{\varepsilon}^{f}(t) z_{1}$ and $S_{\varepsilon}(t) z_{2}$, respectively. Performing the usual products, we find

$$
\frac{d}{d t}\|\bar{z}\|_{\mathcal{H}_{\varepsilon}^{0}}^{2}+2 \sigma_{0}\left\|\partial_{t} \bar{u}\right\|^{2} \leq 2\left\langle W, \partial_{t} \bar{u}\right\rangle+2\left\langle f, \partial_{t} \bar{u}\right\rangle .
$$

Knowing from Lemma 7.2 that $\|\bar{z}(t)\|_{\mathcal{H}_{\varepsilon}^{0}} \leq C$, and using the properties of $\phi$ and $\sigma_{\varepsilon}$, it is easy to check that

$$
2\left\langle W, \partial_{t} \bar{u}\right\rangle \leq \sigma_{0}\left\|\partial_{t} \bar{u}\right\|^{2}+C\|\nabla \bar{u}\|^{2}+C\left\|\nabla \partial_{t} u^{2}\right\|^{2}\|\nabla \bar{u}\|^{2} .
$$


Therefore, we end up with the differential inequality

$$
\frac{d}{d t}\|\bar{z}\|_{\mathcal{H}_{\varepsilon}^{0}}^{2} \leq C\|\bar{z}\|_{\mathcal{H}_{\varepsilon}^{0}}^{2}+C\left\|\nabla \partial_{t} u^{2}\right\|^{2}\|\bar{z}\|_{\mathcal{H}_{\varepsilon}^{0}}^{2}+C\|f\|^{2} .
$$

Since Corollary 8.6 provides the integral estimate for $u^{2}$,

$$
\int_{0}^{t}\left\|\nabla \partial_{t} u^{2}(\tau)\right\|^{2} d \tau \leq \mathcal{Q}(R)(1+t)
$$

the conclusion follows from the Gronwall lemma.

10. Exponential asymptotic smoothing. We now prove the existence of a compact subset of $\mathcal{H}_{\varepsilon}^{0}$, which is exponentially attracting for the semigroup $S_{\varepsilon}(t)$.

Theorem 10.1. There exists $R_{\star}>0$ such that the closed ball

$$
\mathbb{B}_{\varepsilon}^{\star}=\left\{z \in \mathcal{Z}_{\varepsilon}:\|z\|_{\mathcal{Z}_{\varepsilon}} \leq R_{\star}\right\}
$$

contains $\mathbb{B}_{\varepsilon}^{1}$ and is exponentially attracting for $S_{\varepsilon}(t)$. Namely, there is $\nu_{\star}>0$ such that

$$
\boldsymbol{\delta}_{\mathcal{H}_{\varepsilon}^{0}}\left(S_{\varepsilon}(t) \mathbb{B}, \mathbb{B}_{\varepsilon}^{\star}\right) \leq \mathcal{Q}\left(\|\mathbb{B}\|_{\mathcal{H}_{\varepsilon}^{0}}\right) e^{-\nu_{\star} t},
$$

for every bounded set $\mathbb{B} \subset \mathcal{H}_{\varepsilon}^{0}$.

Remark 10.2. From Proposition 8.1, the set $\mathbb{B}_{\varepsilon}^{\star}$ is closed in $\mathcal{H}_{\varepsilon}^{0}$.

We shall make use of the following lemma devised in [9, stating the transitivity of the exponential attraction property 2

Lemma 10.3. Let $S(t)$ be a semigroup on a Banach space $\mathcal{H}$. Let $\mathbb{B}^{0}, \mathbb{B}^{1}, \mathbb{B}^{2} \subset \mathcal{H}$ be such that

$$
\boldsymbol{\delta}_{\mathcal{H}}\left(S(t) \mathbb{B}^{0}, \mathbb{B}^{1}\right) \leq M_{1} e^{-\vartheta_{1} t}, \quad \boldsymbol{\delta}_{\mathcal{H}}\left(S(t) \mathbb{B}^{1}, \mathbb{B}^{2}\right) \leq M_{2} e^{-\vartheta_{2} t},
$$

for some $\vartheta_{1}, \vartheta_{2}>0$ and $M_{1}, M_{2} \geq 0$. Assume also that, for all

$$
z \in \bigcup_{t \geq 0} S(t) \mathbb{B}_{i}, \quad \zeta \in \mathbb{B}_{i+1}, \quad(i=0,1),
$$

the inequality

$$
\|S(t) z-S(t) \zeta\|_{\mathcal{H}} \leq M_{0} e^{\vartheta_{0} t}\|z-\zeta\|_{\mathcal{H}}
$$

holds for some $M_{0} \geq 0$ and $\vartheta_{0} \geq 0$. Then, it follows that

$$
\boldsymbol{\delta}_{\mathcal{H}}\left(S(t) \mathbb{B}^{0}, \mathbb{B}^{2}\right) \leq\left(M_{0} M_{1}+M_{2}\right) e^{-\vartheta t},
$$

where $\vartheta=\frac{\vartheta_{1} \vartheta_{2}}{\vartheta_{0}+\vartheta_{1}+\vartheta_{2}}$.

Proof of Theorem 10.1. In view of Lemma 7.2 and Lemma 10.3, it suffices to prove the existence of a bounded subset of $\mathcal{Z}_{\varepsilon}$ attracting exponentially fast any trajectory originating from the absorbing set $\mathbb{B}_{\varepsilon}^{0}$ of Proposition 7.1. Precisely, we will show that any solution departing from $z \in \mathbb{B}_{\varepsilon}^{0}$ can be decomposed into the sum

$$
S_{\varepsilon}(t) z=S_{\varepsilon}^{1}(t) z+S_{\varepsilon}^{2}(t) z
$$

\footnotetext{
2 In fact, the analogous statement in [9] is slightly less general. But a closer look at its proof shows that our hypotheses suffice to reach the desired conclusion.
} 
where

$$
\begin{aligned}
\left\|S_{\varepsilon}^{1}(t) z\right\|_{\mathcal{H}_{\varepsilon}^{0}} & \leq C e^{-\nu t}, \\
\left\|S_{\varepsilon}^{2}(t) z\right\|_{\mathcal{Z}_{\varepsilon}} & \leq C,
\end{aligned}
$$

for some $\nu>0$ and some $C \geq 0$, depending only on the size of $\mathbb{B}_{\varepsilon}^{0}$.

To this aim, we define

$$
\phi_{0}(r)=\phi(r)+\varpi r,
$$

for some $\varpi>\ell\left(\right.$ note that $\left.\phi_{0}^{\prime} \geq 0\right)$ large enough such that (cf. (2.3) $)$

$$
\frac{1}{2}\|\nabla v\|^{2}+(\varpi-2 \ell)\|v\|^{2}-\left\langle\phi^{\prime}(u(t)) v, v\right\rangle \geq 0, \quad \forall v \in H^{1} .
$$

Then, we choose

$$
S_{\varepsilon}^{1}(t) z=\left(v(t), \partial_{t} v(t), \psi^{t}\right) \quad \text { and } \quad S_{\varepsilon}^{2}(t) z=\left(w(t), \partial_{t} w(t), \xi^{t}\right),
$$

where

$$
\left\{\begin{array}{l}
\varepsilon \partial_{t t} v+\sigma_{\varepsilon}(u) \partial_{t} v-\beta \Delta v-\int_{0}^{\infty} \mu_{\varepsilon}(s) \Delta \psi(s) d s \\
\quad+\left[\sigma_{\varepsilon}(u)-\sigma_{\varepsilon}(w)\right] \partial_{t} w+\beta\left[\phi_{0}(u)-\phi_{0}(w)\right]=0 \\
\partial_{t} \psi=T_{\varepsilon} \psi+\partial_{t} v \\
\left(v(0), \partial_{t} v(0), \psi^{0}\right)=z
\end{array}\right.
$$

and

$$
\left\{\begin{array}{l}
\varepsilon \partial_{t t} w+\sigma_{\varepsilon}(w) \partial_{t} w-\beta \Delta w-\int_{0}^{\infty} \mu_{\varepsilon}(s) \Delta \xi(s) d s+\beta \phi_{0}(w)=\varpi \beta u \\
\partial_{t} \xi=T_{\varepsilon} \xi+\partial_{t} w \\
\left(w(0), \partial_{t} w(0), \xi^{0}\right)=(0,0,0)
\end{array}\right.
$$

The proof of (10.1) -10.2) recasts similar proofs of Lemma 4.1 and Lemma 4.2 in [36]. For the reader's convenience, we sketch here the main steps, pointing out the differences occurring in our case. Observe first that, arguing for (10.3) exactly as in Lemma 7.2, we easily obtain the boundedness of $\left\|S_{\varepsilon}^{2}(t) z\right\|_{\mathcal{H}_{\varepsilon}^{0}}$, along with the integral control

$$
\int_{s}^{t}\left\|\partial_{t} w(\tau)\right\|^{2} d \tau \leq \omega(t-s)+\frac{C}{\omega}, \quad \forall t \geq s>0,
$$

for any $\omega \in(0,1)$. Then, a repetition of the proof for Lemma 8.5, exploiting (17.1) and (10.5), entails the uniform boundedness of $\left\|S_{\varepsilon}^{2}(t) z\right\|_{\mathcal{H}_{\varepsilon}^{1}}$, which, in turn, allows us to apply Lemma 8.7, thus proving (10.2). We also obtain, for every $\omega \in(0,1)$, the estimate

$$
\int_{s}^{t}\left\|\nabla \partial_{t} w(\tau)\right\|^{2} d \tau \leq \omega(t-s)+\frac{C}{\omega}, \quad \forall t \geq s \geq 0 .
$$

In order to prove (10.1), we introduce the functional

$$
\begin{aligned}
\Lambda(t)= & \left\|S_{\varepsilon}^{1}(t) z\right\|_{\mathcal{H}_{\varepsilon}^{0}}^{2}+2 \beta\left\langle\phi_{0}(u(t))-\phi_{0}(w(t)), v\right\rangle \\
& -\beta\left\langle\phi_{0}^{\prime}(u(t)) v(t), v(t)\right\rangle+2 \omega \varepsilon\left\langle v(t), \partial_{t} v(t)\right\rangle .
\end{aligned}
$$


Here, $\omega \in\left(0, \sigma_{0} / 2\right)$ is chosen small enough such that

$$
\frac{1}{4}\left\|S_{\varepsilon}^{1}(t) z\right\|_{\mathcal{H}_{\varepsilon}^{0}}^{2} \leq \Lambda(t) \leq C\left\|S_{\varepsilon}^{1}(t) z\right\|_{\mathcal{H}_{\varepsilon}^{0}}^{2} .
$$

Performing the usual products in (10.4), $\Lambda$ is readily seen to satisfy the differential inequality

$$
\begin{aligned}
& \frac{d}{d t} \Lambda+\omega \Lambda+\frac{\omega \beta}{2}\|\nabla v\|^{2}+\sigma_{0}\left\|\partial_{t} v\right\|^{2}+\frac{\delta}{2 \varepsilon}\|\psi\|_{\mathcal{M}_{\varepsilon}^{0}}^{2}+2 \omega \beta\left\langle\phi_{0}(u)-\phi_{0}(w), v\right\rangle \\
& \leq-2 \omega\langle\psi, v\rangle_{\mathcal{M}_{\varepsilon}^{0}}-2\left\langle\left[\sigma_{\varepsilon}(u)-\sigma_{\varepsilon}(w)\right] \partial_{t} w, \partial_{t} v\right\rangle-2 \omega\left\langle\sigma_{\varepsilon}(u) \partial_{t} v, v\right\rangle \\
& \quad-2 \omega\left\langle\left[\sigma_{\varepsilon}(u)-\sigma_{\varepsilon}(w)\right] \partial_{t} w, v\right\rangle+2 \beta\left\langle\left[\phi_{0}^{\prime}(u)-\phi_{0}^{\prime}(w)\right] \partial_{t} w, v\right\rangle-\beta\left\langle\phi_{0}^{\prime \prime}(u) \partial_{t} u, v^{2}\right\rangle .
\end{aligned}
$$

The first two terms on the right-hand side are estimated as

$$
\begin{aligned}
& -2 \omega\langle\psi, v\rangle_{\mathcal{M}_{\varepsilon}^{0}}-2\left\langle\left[\sigma_{\varepsilon}(u)-\sigma_{\varepsilon}(w)\right] \partial_{t} w, \partial_{t} v\right\rangle \\
& \leq \frac{\omega \beta}{6}\|\nabla v\|^{2}+\frac{\delta}{2 \varepsilon}\|\psi\|_{\mathcal{M}_{\varepsilon}^{0}}^{2}+C\left\|\nabla \partial_{t} w\right\|\|\nabla v\|\left\|\partial_{t} v\right\| \\
& \leq \frac{\omega \beta}{6}\|\nabla v\|^{2}+\frac{\sigma_{0}}{2}\left\|\partial_{t} v\right\|^{2}+\frac{\delta}{2 \varepsilon}\|\psi\|_{\mathcal{M}_{\varepsilon}^{0}}^{2}+C\left\|\nabla \partial_{t} w\right\|^{2} \Lambda,
\end{aligned}
$$

whereas the remaining terms, arguing exactly as in [36], are controlled by

$$
\frac{\omega}{2} \Lambda+\frac{\omega \beta}{3}\|\nabla v\|^{2}+\frac{\sigma_{0}}{2}\left\|\partial_{t} v\right\|^{2}+C\left(\left\|\partial_{t} u\right\|^{2}+\left\|\nabla \partial_{t} w\right\|^{2}\right) \Lambda .
$$

Hence, fixing $\omega$ suitably small, we are led to the differential inequality

$$
\frac{d}{d t} \Lambda+\omega \Lambda \leq C\left(\left\|\partial_{t} u\right\|^{2}+\left\|\nabla \partial_{t} w\right\|^{2}\right) \Lambda .
$$

The dissipation integrals (17.1) and (10.6) allow us to apply Lemma 8.3, which yields

$$
\Lambda(t) \leq C \Lambda(0) e^{-\frac{\omega t}{2}} .
$$

This finishes the proof of (10.1).

11. Estimate of the difference of trajectories. The aim of this section is to compare the trajectories of $S_{\varepsilon}(t)$ and $S_{0}(t)$ originating from the same initial position $u_{0} \in H^{2}$, providing a quantitative estimate of their difference on finite time intervals as $\varepsilon$ tends to zero. Given $\varepsilon \in(0,1]$ and $z=\left(u_{0}, v_{0}, \eta_{0}\right)$, we denote

$$
S_{\varepsilon}(t) z=\left(u(t), \partial_{t} u(t), \eta^{t}\right) \quad \text { and } \quad S_{0}(t)\left(u_{0}, 0,0\right)=(\hat{u}(t), 0,0) .
$$

Theorem 11.1. Let $a<1 / 4$ be fixed. Then, for all $\varepsilon \in(0,1]$ and $z=\left(u_{0}, v_{0}, \eta_{0}\right) \in \mathcal{H}_{\varepsilon}^{1}$, with $\eta_{0} \in \operatorname{dom}\left(T_{\varepsilon}\right)$ satisfying

$$
\|z\|_{\mathcal{H}_{\varepsilon}^{1}}+\varepsilon\left\|T_{\varepsilon} \eta_{0}\right\|_{\mathcal{M}_{\varepsilon}^{0}} \leq R
$$

the following estimates hold:

$$
\begin{gathered}
\|\nabla u(t)-\nabla \hat{u}(t)\| \leq \mathcal{Q}(R) e^{\mathcal{Q}(R) t} \varepsilon^{a}, \\
\varepsilon\left\|\partial_{t} u(t)\right\|^{2}+\left\|\eta^{t}\right\|_{\mathcal{M}_{\varepsilon}^{0}}^{2} \leq \mathcal{Q}(R)\left[e^{-\frac{\varrho t}{\sqrt{\varepsilon}}}+\varepsilon\right],
\end{gathered}
$$

for some $\varrho>0$. 
REMARK 11.2. For further scopes, we define the operator $\Pi: \mathcal{H}_{\varepsilon}^{0} \rightarrow \mathcal{H}_{0}^{0}$ as

$$
\Pi\left(u_{0}, v_{0}, \eta_{0}\right)=\left(u_{0}, 0,0\right) .
$$

Then, setting any $t^{\star}>0$, and collecting (11.1)-(11.2), we obtain the estimate

$$
\left\|S_{\varepsilon}(t) z-S_{0}(t) \Pi z\right\|_{\mathcal{H}_{\varepsilon}^{0}} \leq \mathcal{Q}(R) e^{\mathcal{Q}(R) t} \varepsilon^{a}, \quad \forall t \geq t^{\star} .
$$

We first note that, in light of Remark 7.3, inequalities (11.1)-(11.2) need to be proved only when $\varepsilon$ is close to zero. Accordingly, we assume without loss of generality $\varepsilon \in\left(0, \varepsilon_{0}\right]$, with $\varepsilon_{0}$ as in (5.6). Let then $z$ fulfill the assumptions of the theorem. Until the end of the section, the generic constant $C \geq 0$ may depend (increasingly) on $R$.

The proof of Theorem 11.1 will be carried out through several lemmas, where, on account of Lemma 8.5, we will exploit the uniform bounds

$$
\|\Delta \hat{u}\|+\|\Delta u\|+\sqrt{\varepsilon}\left\|\nabla \partial_{t} u\right\|+\|\eta\|_{\mathcal{M}_{\varepsilon}^{1}} \leq C,
$$

which, in particular, imply

$$
\left\|\phi^{\prime}(\hat{u})\right\|_{L^{\infty}}+\left\|\phi^{\prime}(u)\right\|_{L^{\infty}}+\left\|\phi^{\prime \prime}(u)\right\|_{L^{\infty}}+\left\|\sigma_{\varepsilon}(u)\right\|_{L^{\infty}} \leq C .
$$

LEMMA 11.3. We have the inequality

$$
\left\|\eta^{t}\right\|_{\mathcal{M}_{\varepsilon}^{0}}^{2} \leq\left\|\eta_{0}\right\|_{\mathcal{M}_{\varepsilon}^{0}}^{2} e^{-\frac{\delta t}{2 \varepsilon}}+C \varepsilon .
$$

Proof. Multiplying the second equation of (6.3) by $\eta$ in $\mathcal{M}_{\varepsilon}^{0}$, and using (6.1), we obtain

$$
\frac{d}{d t}\|\eta\|_{\mathcal{M}_{\varepsilon}^{0}}^{2}+\frac{\delta}{\varepsilon}\|\eta\|_{\mathcal{M}_{\varepsilon}^{0}}^{2} \leq 2\left\|\nabla \partial_{t} u\right\| \int_{0}^{\infty} \mu_{\varepsilon}(s)\|\nabla \eta(s)\| d s \leq \frac{C}{\sqrt{\varepsilon}}\|\eta\|_{\mathcal{M}_{\varepsilon}^{0}} \leq \frac{\delta}{2 \varepsilon}\|\eta\|_{\mathcal{M}_{\varepsilon}^{0}}^{2}+C .
$$

An application of the Gronwall lemma completes the argument.

LEMma 11.4. There exists $\varrho>0$ such that

$$
\varepsilon\left\|\partial_{t} u(t)\right\|^{2} \leq C e^{-\frac{\varrho t}{\sqrt{\varepsilon}}}+C \varepsilon
$$

Proof. Setting $v=\partial_{t} u$ and $\xi=\partial_{t} \eta$, we differentiate (6.3) with respect to time. Noting that $\sigma_{\varepsilon}^{\prime}=\varepsilon \phi^{\prime \prime}$, this leads to

$$
\left\{\begin{array}{l}
\varepsilon \partial_{t t} v+\sigma_{\varepsilon}(u) \partial_{t} v+\varepsilon \phi^{\prime \prime}(u) v^{2}-\beta \Delta v-\int_{0}^{\infty} \mu_{\varepsilon}(s) \Delta \xi(s) d s+\beta \phi^{\prime}(u) v=0, \\
\partial_{t} \xi=T_{\varepsilon} \xi+\partial_{t} v
\end{array}\right.
$$

For $\omega \in\left(0, \sigma_{0} / 2\right)$, we multiply the first equation by $\partial_{t} v+\frac{\omega}{\sqrt{\varepsilon}} v$ in $H^{0}$, and the second one by $\xi$ in $\mathcal{M}_{\varepsilon}^{0}$. Recalling (5.7) and (6.1), we find

$$
\begin{aligned}
& \frac{d}{d t} \Lambda+\frac{2 \omega \beta}{\sqrt{\varepsilon}}\|\nabla v\|^{2}+\sigma_{0}\left\|\partial_{t} v\right\|^{2}+\frac{\delta}{\varepsilon}\|\xi\|_{\mathcal{M}_{\varepsilon}^{0}}^{2} \\
& \leq-2 \varepsilon\left\langle\phi^{\prime \prime}(u) v^{2}, \partial_{t} v\right\rangle-2 \beta\left\langle\phi^{\prime}(u) v, \partial_{t} v\right\rangle-\frac{2 \omega}{\sqrt{\varepsilon}}\left\langle\sigma_{\varepsilon}(u) \partial_{t} v, v\right\rangle-2 \omega \sqrt{\varepsilon}\left\langle\phi^{\prime \prime}(u) v^{2}, v\right\rangle \\
& \quad-\frac{2 \omega \beta}{\sqrt{\varepsilon}}\left\langle\phi^{\prime}(u) v, v\right\rangle-\frac{2 \omega}{\sqrt{\varepsilon}}\langle\xi, v\rangle_{\mathcal{M}_{\varepsilon}^{0}},
\end{aligned}
$$

where we set

$$
\Lambda(t)=\beta\|\nabla v(t)\|^{2}+\varepsilon\left\|\partial_{t} v(t)\right\|^{2}+\left\|\xi^{t}\right\|_{\mathcal{M}_{\varepsilon}^{0}}^{2}+2 \omega \sqrt{\varepsilon}\left\langle\partial_{t} v(t), v(t)\right\rangle .
$$


It is clear that, if $\omega$ is small enough,

$$
\frac{1}{2} \Lambda(t) \leq \beta\|\nabla v(t)\|^{2}+\varepsilon\left\|\partial_{t} v(t)\right\|^{2}+\left\|\xi^{t}\right\|_{\mathcal{M}_{\varepsilon}^{0}}^{2} \leq 2 \Lambda(t) .
$$

We now estimate the terms on the right-hand side of the differential inequality. Exploiting (2.1) and the fact that $\varepsilon\|\nabla v\| \leq C$, we have

$$
-2 \varepsilon\left\langle\phi^{\prime \prime}(u) v^{2}, \partial_{t} v\right\rangle-2 \beta\left\langle\phi^{\prime}(u) v, \partial_{t} v\right\rangle \leq C\|v\|\left\|\partial_{t} v\right\| \leq \frac{\sigma_{0}}{4}\left\|\partial_{t} v\right\|^{2}+C\|v\|^{2}
$$

and

$$
\begin{aligned}
& -\frac{2 \omega}{\sqrt{\varepsilon}}\left(\left\langle\sigma_{\varepsilon}(u) \partial_{t} v, v\right\rangle+\varepsilon\left\langle\phi^{\prime \prime}(u) v^{2}, v\right\rangle+\beta\left\langle\phi^{\prime}(u) v, v\right\rangle\right) \\
& \leq \frac{C}{\sqrt{\varepsilon}}\left(\|v\|\left\|\partial_{t} v\right\|+\varepsilon\|\nabla v\|\|v\|^{2}+\|v\|^{2}\right) \\
& \leq \frac{\sigma_{0}}{4}\left\|\partial_{t} v\right\|^{2}+\frac{C}{\varepsilon}\|v\|^{2} .
\end{aligned}
$$

Finally, provided that $\omega$ is small enough,

$$
-\frac{2 \omega}{\sqrt{\varepsilon}}\langle\xi, v\rangle_{\mathcal{M}_{\varepsilon}^{0}} \leq \frac{\delta}{2 \varepsilon}\|\xi\|_{\mathcal{M}_{\varepsilon}^{0}}^{2}+\frac{\omega \beta}{\sqrt{\varepsilon}}\|\nabla v\|^{2} .
$$

Collecting the estimates above, we are led to the inequality

$$
\frac{d}{d t} \Lambda+\frac{\varrho}{\sqrt{\varepsilon}} \Lambda \leq \frac{C}{\varepsilon}\|v\|^{2}
$$

for some $\varrho>0$. In light of the integral control for $\|v\|^{2}$ given by (7.1), the Gronwall lemma yields

Indeed,

$$
\varepsilon\left\|\partial_{t t} u(t)\right\|^{2} \leq 2 \Lambda(t) \leq \frac{C}{\varepsilon^{2}} e^{-\frac{\varrho t}{\sqrt{\varepsilon}}}+\frac{C}{\varepsilon}
$$

$$
\Lambda(0) \leq 2\left(\beta\left\|\nabla v_{0}\right\|^{2}+\varepsilon\left\|\partial_{t t} u(0)\right\|^{2}+\left\|\partial_{t} \eta^{0}\right\|_{\mathcal{M}_{\varepsilon}^{0}}^{2}\right) \leq \frac{C}{\varepsilon^{2}} .
$$

The last inequality follows at once by the assumptions on $z=\left(u_{0}, v_{0}, \eta_{0}\right)$, observing that the initial values of $\partial_{t t} u$ and $\eta$ are read from (6.3) at $t=0$, namely,

$$
\varepsilon \partial_{t t} u(0)=-\sigma_{\varepsilon}\left(u_{0}\right) v_{0}+\beta \Delta u_{0}-\beta \phi\left(u_{0}\right)+\int_{0}^{\infty} \mu_{\varepsilon}(s) \Delta \eta_{0}(s) d s
$$

and

$$
\partial_{t} \eta^{0}=T_{\varepsilon} \eta_{0}+v_{0}
$$

Then, multiplying the first equation of (6.3) by $\partial_{t} u$ in $H^{0}$, in view of (5.7), we have

$$
\sigma_{0}\left\|\partial_{t} u\right\|^{2} \leq \beta\left\langle\Delta u, \partial_{t} u\right\rangle-\beta\left\langle\phi(u), \partial_{t} u\right\rangle-\varepsilon\left\langle\partial_{t t} u, \partial_{t} u\right\rangle+\int_{0}^{\infty} \mu_{\varepsilon}(s)\left\langle\Delta \eta(s), \partial_{t} u\right\rangle d s .
$$

The right-hand side is controlled by

$$
C\left\|\partial_{t} u\right\|+\varepsilon\left\|\partial_{t t} u\right\|\left\|\partial_{t} u\right\| \leq \frac{\sigma_{0}}{2}\left\|\partial_{t} u\right\|^{2}+C \varepsilon^{2}\left\|\partial_{t t} u\right\|^{2}+C .
$$

Therefore,

$$
\left\|\partial_{t} u\right\|^{2} \leq C \varepsilon^{2}\left\|\partial_{t t} u\right\|^{2}+C .
$$

The desired result follows from (11.5).

A crucial step is a suitable estimate of the norm of $\partial_{t t} u$. 
Lemma 11.5. Let $a<1 / 4$ be fixed. Then,

$$
\varepsilon^{2}\left\|\partial_{t t} u(t)\right\|^{2} \leq C \sqrt{\varepsilon},
$$

whenever $t \geq 2 \varepsilon^{2 a}$.

Proof. With the notation of the preceding lemma, we first observe that (cf. (11.5))

$$
\Lambda(t) \leq \frac{C}{\varepsilon^{2}}
$$

and

$$
\|v(t)\|=\left\|\partial_{t} u(t)\right\| \leq C, \quad \forall t \geq \varepsilon^{2 a} .
$$

Thus, for $t \geq \varepsilon^{2 a}$, the differential inequality (11.4) turns into

$$
\frac{d}{d t} \Lambda+\frac{\varrho}{\sqrt{\varepsilon}} \Lambda \leq \frac{C}{\varepsilon}
$$

and the Gronwall lemma entails

$$
\Lambda(t) \leq \Lambda\left(\varepsilon^{2 a}\right) e^{-\frac{\varrho\left(t-\varepsilon^{2 a}\right)}{\sqrt{\varepsilon}}}+\frac{C}{\sqrt{\varepsilon}} \leq \frac{C}{\varepsilon^{2}} e^{-\frac{\varrho\left(t-\varepsilon^{2 a}\right)}{\sqrt{\varepsilon}}}+\frac{C}{\sqrt{\varepsilon}}, \quad \forall t \geq \varepsilon^{2 a} .
$$

Consequently, if $t \geq 2 \varepsilon^{2 a}$,

$$
\varepsilon^{2}\left\|\partial_{t t} u(t)\right\|^{2} \leq 2 \varepsilon \Lambda(t) \leq \frac{C}{\varepsilon} e^{-\frac{\rho \varepsilon^{2 a}}{\sqrt{\varepsilon}}}+C \sqrt{\varepsilon} \leq C \sqrt{\varepsilon}
$$

as $a<1 / 4$.

The next result provides the proof of (11.1).

LEMma 11.6. The inequality

$$
\|\nabla u(t)-\nabla \hat{u}(t)\| \leq C e^{C t} \varepsilon^{a}
$$

holds for every fixed $a<1 / 4$.

Proof. Let $a<1 / 4$ be given. The difference $\bar{u}(t)=u(t)-\hat{u}(t)$ solves the parabolic problem

$$
\left\{\begin{array}{l}
\beta \partial_{t} \bar{u}-\beta \Delta \bar{u}=\int_{0}^{\infty} \mu_{\varepsilon}(s) \Delta \eta(s) d s-\varepsilon \partial_{t t} u-\beta[\phi(u)-\phi(\hat{u})]-\varepsilon \phi^{\prime}(u) \partial_{t} u, \\
\bar{u}(0)=0
\end{array}\right.
$$

Assume first that $t \geq 2 \varepsilon^{2 a}$. The product by $\partial_{t} \bar{u}$ in $H^{0}$ entails the equality

$$
\begin{aligned}
& \frac{d}{d t} \beta\|\nabla \bar{u}\|^{2}+2 \beta\left\|\partial_{t} \bar{u}\right\|^{2} \\
& =-2\left\langle\eta, \partial_{t} \bar{u}\right\rangle_{\mathcal{M}_{\varepsilon}^{0}}-2 \varepsilon\left\langle\partial_{t t} u, \partial_{t} \bar{u}\right\rangle-2 \beta\left\langle\phi(u)-\phi(\hat{u}), \partial_{t} \bar{u}\right\rangle-2 \varepsilon\left\langle\phi^{\prime}(u) \partial_{t} u, \partial_{t} \bar{u}\right\rangle .
\end{aligned}
$$

The memory term on the right-hand side is easily estimated by Lemma 11.3 as

$$
-2\left\langle\eta, \partial_{t} \bar{u}\right\rangle_{\mathcal{M}_{\varepsilon}^{0}} \leq C\left\|\nabla \partial_{t} \bar{u}\right\|\|\eta\|_{\mathcal{M}_{\varepsilon}^{0}} \leq \sqrt{\varepsilon}\left\|\nabla \partial_{t} \bar{u}\right\|^{2}+C \sqrt{\varepsilon} .
$$

Using Lemma 11.5, we deduce that

$$
-2 \varepsilon\left\langle\partial_{t t} u, \partial_{t} \bar{u}\right\rangle \leq C \varepsilon^{2}\left\|\partial_{t t} u\right\|^{2}+\beta\left\|\partial_{t} \bar{u}\right\|^{2} \leq C \sqrt{\varepsilon}+\beta\left\|\partial_{t} \bar{u}\right\|^{2} .
$$


Since $\varepsilon\left\|\partial_{t} u\right\|^{2} \leq C$, we have

$$
\begin{aligned}
-2 \beta\left\langle\phi(u)-\phi(\hat{u}), \partial_{t} \bar{u}\right\rangle-2 \varepsilon\left\langle\phi^{\prime}(u) \partial_{t} u, \partial_{t} \bar{u}\right\rangle & \leq C\left(\|\bar{u}\|+\varepsilon\left\|\partial_{t} u\right\|\right)\left\|\partial_{t} \bar{u}\right\| \\
& \leq C\|\nabla \bar{u}\|^{2}+C \varepsilon+\beta\left\|\partial_{t} \bar{u}\right\|^{2} .
\end{aligned}
$$

In conclusion, we obtain

where we set

$$
\frac{d}{d t}\|\nabla \bar{u}\|^{2} \leq C\|\nabla \bar{u}\|^{2}+g
$$

From Corollary 8.6 ,

$$
g(t)=\frac{\sqrt{\varepsilon}}{\beta}\left\|\nabla \partial_{t} \bar{u}(t)\right\|^{2}+C \sqrt{\varepsilon} .
$$

$$
\int_{0}^{t}\left\|\nabla \partial_{t} \bar{u}(\tau)\right\|^{2} d \tau \leq C(1+t)
$$

Therefore, for every $t \geq 2 \varepsilon^{2 a}$, the Gronwall lemma gives

$$
\|\nabla \bar{u}(t)\|^{2} \leq C e^{C t}\left(\left\|\nabla \bar{u}\left(2 \varepsilon^{2 a}\right)\right\|^{2}+\sqrt{\varepsilon}\right) .
$$

On the other hand, if $t \leq 2 \varepsilon^{2 a}$, we have

$$
\|\nabla \bar{u}(t)\|^{2} \leq\left(\int_{0}^{t}\left\|\nabla \partial_{t} \bar{u}(\tau)\right\| d \tau\right)^{2} \leq 2 \varepsilon^{2 a} \int_{0}^{1}\left\|\nabla \partial_{t} \bar{u}(\tau)\right\|^{2} d \tau \leq C \varepsilon^{2 a} .
$$

Thus, the sought inequality is verified for every $t \geq 0$.

12. Robust exponential attractors. The main result concerning the asymptotic properties of $S_{\varepsilon}(t)$ is the existence of a family of exponential attractors $\mathcal{E}_{\varepsilon}$, which is robust (in an appropriate sense) with respect to the singular limit $\varepsilon \rightarrow 0$.

Theorem 12.1. For every $\varepsilon \in\left[0, \varepsilon_{0}\right]$, there exists a compact set $\mathcal{E}_{\varepsilon}$, called exponential attractor, which is compact in $\mathcal{H}_{\varepsilon}^{0}$ and bounded in $\mathcal{Z}_{\varepsilon}$, and satisfies the following properties:

(I) The fractal dimension of $\mathcal{E}_{\varepsilon}$ in $\mathcal{H}_{\varepsilon}^{0}$ is uniformly bounded with respect to $\varepsilon$.

(II) The set $\mathcal{E}_{\varepsilon}$ attracts any bounded subset $\mathbb{B} \subset \mathcal{H}_{\varepsilon}^{0}$ with an exponential rate which is uniform with respect to $\varepsilon$; namely, there exists $\kappa>0$ (independent of $\varepsilon$ and of the choice of $\mathbb{B}$ ) such that

$$
\boldsymbol{\delta}_{\mathcal{H}_{\varepsilon}^{0}}\left(S_{\varepsilon}(t) \mathbb{B}, \mathcal{E}_{\varepsilon}\right) \leq \mathcal{Q}\left(\|\mathbb{B}\|_{\mathcal{H}_{\varepsilon}^{0}}\right) e^{-\kappa t} .
$$

(III) There exists $\tau>0$ such that

$$
\boldsymbol{\delta}_{\mathcal{H}_{\varepsilon}^{0}}^{\mathrm{sym}}\left(\mathcal{E}_{\varepsilon}, \mathcal{E}_{0}\right) \leq C \varepsilon^{\tau} .
$$

Moreover, $\mathcal{E}_{\varepsilon}$ is positively invariant for $S_{\varepsilon}(t)$; that is, $S_{\varepsilon}(t) \mathcal{E}_{\varepsilon} \subset \mathcal{E}_{\varepsilon}$.

This theorem is proved combining the abstract theorems of [6, 14. Indeed, in the present model, we actually have to deal with a double singular limit (one on the velocity and one on the memory component). To this end, we take the exponentially attracting set $\mathbb{B}_{\varepsilon}^{\star}$ of Theorem 10.1, and we choose $t^{\star}>0$ large enough such that

$$
S_{\varepsilon}=S_{\varepsilon}\left(t^{\star}\right): \mathbb{B}_{\varepsilon}^{\star} \rightarrow \mathbb{B}_{\varepsilon}^{\star} .
$$


In view of the transitivity of the exponential attraction property provided by Lemma 10.3 . we have the following result (see [6, 14]).

LEMma 12.2. Assume that the following hold.

(H1) For some $\lambda \in\left[0, \frac{1}{2}\right)$ and for every $z_{1}, z_{2} \in \mathbb{B}_{\varepsilon}^{\star}$, we have

$$
S_{\varepsilon} z_{1}-S_{\varepsilon} z_{2}=D_{\varepsilon}\left(z_{1}, z_{2}\right)+K_{\varepsilon}\left(z_{1}, z_{2}\right),
$$

where

$$
\begin{aligned}
\left\|D_{\varepsilon}\left(z_{1}, z_{2}\right)\right\|_{\mathcal{H}_{\varepsilon}^{0}} & \leq \lambda\left\|z_{1}-z_{2}\right\|_{\mathcal{H}_{\varepsilon}^{0}}, \\
\left\|K_{\varepsilon}\left(z_{1}, z_{2}\right)\right\|_{\mathcal{Z}_{\varepsilon}} & \leq C\left\|z_{1}-z_{2}\right\|_{\mathcal{H}_{\varepsilon}^{0}} .
\end{aligned}
$$

(H2) For some $a>0$, every $t \geq t^{\star}$ and every $z \in \mathbb{B}_{\varepsilon}^{\star}$,

$$
\left\|S_{\varepsilon}(t) z-S_{0}(t) \Pi z\right\|_{\mathcal{H}_{\varepsilon}^{0}} \leq C e^{C t} \varepsilon^{a} .
$$

(H3) For every $t \in\left[t^{\star}, 2 t^{\star}\right]$, the map

$$
z \mapsto S_{\varepsilon}(t) z: \mathbb{B}_{\varepsilon}^{\star} \rightarrow \mathbb{B}_{\varepsilon}^{\star}
$$

is Lipschitz continuous in the topology of $\mathcal{H}_{\varepsilon}^{0}$, with a Lipschitz constant independent of $t \in\left[t^{\star}, 2 t^{\star}\right]$ and of $\varepsilon$.

(H4) For every $\varepsilon \in\left[0, \varepsilon_{0}\right]$ and every $z \in \mathbb{B}_{\varepsilon}^{\star}$, the map

$$
t \mapsto S_{\varepsilon}(t) z:\left[t^{\star}, 2 t^{\star}\right] \rightarrow \mathbb{B}_{\varepsilon}^{\star}
$$

is $\frac{1}{2}$-Hölder continuous, with a Hölder constant that may depend on $\varepsilon$, but is independent of $z \in \mathbb{B}_{\varepsilon}^{\star}$. Again, $\mathbb{B}_{\varepsilon}^{\star}$ is endowed with the topology of $\mathcal{H}_{\varepsilon}^{0}$.

Then, the conclusions of Theorem 12.1 hold true.

Proof of Theorem 12.1. In light of Proposition 9.1 and Remark 11.2, conditions (H2)(H3) of Lemma 12.2 are already proven, while (H4) is a direct consequence of the bounds on the time-derivatives. Indeed, assume that $2 t^{\star} \geq t_{1}>t_{2} \geq t^{\star}$. Then, appealing to Corollary 8.6, we obtain

$$
\left\|\nabla u\left(t_{1}\right)-\nabla u\left(t_{2}\right)\right\| \leq \int_{t_{2}}^{t_{1}}\left\|\nabla \partial_{t} u(\tau)\right\| d \tau \leq C \sqrt{t_{1}-t_{2}},
$$

while, if $\varepsilon>0$, we deduce from the proof of Lemma 11.5 that

$$
\begin{aligned}
\sqrt{\varepsilon}\left\|\partial_{t} u\left(t_{1}\right)-\partial_{t} u\left(t_{2}\right)\right\|+\left\|\eta^{t_{1}}-\eta^{t_{2}}\right\|_{\mathcal{M}_{\varepsilon}^{0}} & \leq \int_{t_{2}}^{t_{1}}\left[\sqrt{\varepsilon}\left\|\partial_{t t} u(\tau)\right\|+\left\|\partial_{t} \eta^{\tau}\right\|_{\mathcal{M}_{\varepsilon}^{0}}\right] d \tau \\
& \leq \frac{C}{\sqrt[4]{\varepsilon}}\left(t_{1}-t_{2}\right)
\end{aligned}
$$

Thus, exploiting (11.2), it is possible to rewrite the latter estimate as

$$
\sqrt{\varepsilon}\left\|\partial_{t} u\left(t_{1}\right)-\partial_{t} u\left(t_{2}\right)\right\|+\left\|\eta^{t_{1}}-\eta^{t_{2}}\right\|_{\mathcal{M}_{\varepsilon}^{0}} \leq C \sqrt{t_{1}-t_{2}} .
$$

In summary, (H4) holds, even with a Hölder constant independent of $\varepsilon$. In order to complete the proof of Theorem 12.1, we are left to show the validity of (H1). To this 
aim, for $z_{i} \in \mathbb{B}_{\varepsilon}^{\star}$, we denote $S_{\varepsilon}(t) z_{i}=\left(u^{i}(t), \partial_{t} u^{i}(t), \eta^{i t}\right)$, and we set

$$
\Psi(t)=\frac{1}{\varepsilon} \frac{\sigma_{\varepsilon}\left(u^{1}(t)\right)-\sigma_{\varepsilon}\left(u^{2}(t)\right)}{u^{1}(t)-u^{2}(t)}=\int_{0}^{1} \phi^{\prime \prime}\left(s u^{1}(t)+(1-s) u^{2}(t)\right) d s .
$$

At this point, we exploit the decomposition devised in [15], namely,

$$
S_{\varepsilon}(t) z_{1}-S_{\varepsilon}(t) z_{2}=\zeta^{1}(t)+\zeta^{2}(t)
$$

where

$$
\zeta^{1}(t)=\left(v(t), \partial_{t} v(t), \psi^{t}\right) \quad \text { and } \quad \zeta^{2}(t)=\left(w(t), \partial_{t} w(t), \xi^{t}\right)
$$

solve the problems

$$
\left\{\begin{array}{l}
\varepsilon \partial_{t t} v-\beta \Delta v-\int_{0}^{\infty} \mu_{\varepsilon}(s) \Delta \psi(s) d s+\sigma_{\varepsilon}\left(u^{1}\right) \partial_{t} v+\varepsilon \Psi v \partial_{t} u^{2}=0 \\
\partial_{t} \psi=T_{\varepsilon} \psi+\partial_{t} v \\
\zeta^{1}(0)=z_{1}-z_{2}
\end{array}\right.
$$

and

$$
\left\{\begin{array}{l}
\varepsilon \partial_{t t} w-\beta \Delta w-\int_{0}^{\infty} \mu_{\varepsilon}(s) \Delta \xi(s) d s+\sigma_{\varepsilon}\left(u^{1}\right) \partial_{t} w+\varepsilon \Psi w \partial_{t} u^{2}+\beta \phi\left(u^{1}\right)-\beta \phi\left(u^{2}\right)=0 \\
\partial_{t} \xi=T_{\varepsilon} \xi+\partial_{t} w \\
\zeta^{2}(0)=0 .
\end{array}\right.
$$

Setting $\omega \in\left(0, \sigma_{0} / 2\right)$ small enough such that

$$
\frac{1}{2}\left\|\zeta^{1}(t)\right\|_{\mathcal{H}_{\varepsilon}^{0}}^{2} \leq \Lambda(t)=\left\|\zeta^{1}(t)\right\|_{\mathcal{H}_{\varepsilon}^{0}}^{2}+2 \omega \varepsilon\left\langle\partial_{t} v(t), v(t)\right\rangle \leq 2\left\|\zeta^{1}(t)\right\|_{\mathcal{H}_{\varepsilon}^{0}}^{2},
$$

and making (by now) standard calculations, we arrive at the differential inequality

$$
\begin{aligned}
& \frac{d}{d t} \Lambda+2 \omega \beta\|\nabla v\|^{2}+\sigma_{0}\left\|\partial_{t} v\right\|^{2}+\frac{\delta}{\varepsilon}\|\psi\|_{\mathcal{M}_{\varepsilon}^{0}}^{2} \\
& \leq-2 \varepsilon\left\langle\Psi \partial_{t} u^{2} v, \partial_{t} v\right\rangle-2 \varepsilon \omega\left\langle\Psi \partial_{t} u^{2} v, v\right\rangle-2 \omega\left\langle\sigma_{\varepsilon}\left(u^{1}\right) \partial_{t} v, v\right\rangle-2 \omega\langle\psi, v\rangle_{\mathcal{M}_{\varepsilon}^{0}} .
\end{aligned}
$$

We see that

$$
-2 \varepsilon\left\langle\Psi \partial_{t} u^{2} v, \partial_{t} v\right\rangle-2 \varepsilon \omega\left\langle\Psi \partial_{t} u^{2} v, v\right\rangle \leq C\left\|\partial_{t} u^{2}\right\|^{2} \Lambda+\frac{\omega \beta}{2}\|\nabla v\|^{2}+\frac{\sigma_{0}}{4}\left\|\partial_{t} v\right\|^{2},
$$

and, for $\omega$ small enough,

$$
-2 \omega\left\langle\sigma_{\varepsilon}\left(u^{1}\right) \partial_{t} v, v\right\rangle-2 \omega\langle\psi, v\rangle_{\mathcal{M}_{\varepsilon}^{0}} \leq \frac{\omega \beta}{2}\|\nabla v\|^{2}+\frac{\sigma_{0}}{4}\left\|\partial_{t} v\right\|^{2}+\frac{\delta}{2 \varepsilon}\|\psi\|_{\mathcal{M}_{\varepsilon}^{0}}^{2} .
$$

Thus, we obtain

$$
\frac{d}{d t} \Lambda+\nu \Lambda \leq C\left\|\partial_{t} u^{2}\right\|^{2} \Lambda
$$

for some $\nu>0$. From (7.1) and Lemma 8.3, we conclude that

$$
\left\|\zeta^{1}\left(t^{\star}\right)\right\|_{\mathcal{H}_{\varepsilon}^{0}}<\frac{1}{4}\left\|z_{1}-z_{2}\right\|_{\mathcal{H}_{\varepsilon}^{0}}
$$

provided that we suitably enlarge $t^{\star}$. Similarly, knowing that, as $\phi \in C^{3}(\mathbb{R})$,

$$
\|\nabla \Psi(t)\| \leq C,
$$


we have

$$
\frac{d}{d t}\left\|\zeta^{2}\right\|_{\mathcal{H}_{\varepsilon}^{1}}^{2} \leq C\left\|\zeta^{2}\right\|_{\mathcal{H}_{\varepsilon}^{1}}^{2}+C\left\|\nabla u^{1}-\nabla u^{2}\right\|^{2}
$$

Using the Gronwall lemma, along with the continuous dependence estimate of Proposition 9.1, we end up with

$$
\left\|\zeta^{2}\left(t^{\star}\right)\right\|_{\mathcal{H}_{\varepsilon}^{1}} \leq C\left\|z_{1}-z_{2}\right\|_{\mathcal{H}_{\varepsilon}^{0}} .
$$

Then, an application of Lemma 8.7 yields

$$
\left\|\zeta^{2}\left(t^{\star}\right)\right\|_{\mathcal{Z}_{\varepsilon}} \leq C\left\|z_{1}-z_{2}\right\|_{\mathcal{H}_{\varepsilon}^{0}}
$$

Therefore, condition (H1) with $\lambda=1 / 4$ follows by setting $D_{\varepsilon}\left(z_{1}, z_{2}\right)=\zeta^{1}\left(t^{\star}\right)$ and $K_{\varepsilon}\left(z_{1}, z_{2}\right)=\zeta^{2}\left(t^{\star}\right)$

13. Global attractors and convergence to equilibria. Theorem 12.1 provides, in particular, the existence of a compact attracting set. Therefore, from a classical result in the theory of dynamical systems, we conclude that $S_{\varepsilon}(t)$ has the global attractor (cf. [2, 23, 40]).

TheOREm 13.1. For every $\varepsilon \in\left[0, \varepsilon_{0}\right]$, the semigroup $S_{\varepsilon}(t)$ possesses a global attractor $\mathcal{A}_{\varepsilon} \subset \mathcal{E}_{\varepsilon}$, whose fractal dimension is bounded, uniformly with respect to $\varepsilon$.

REMARK 13.2. Due to the regularity of $\mathcal{A}_{\varepsilon}$, applying the same techniques as in [19, 24], it is not hard to show the upper semicontinuity at $\varepsilon=0$ of the family $\left\{\mathcal{A}_{\varepsilon}\right\}$, namely,

$$
\lim _{\varepsilon \rightarrow 0} \boldsymbol{\delta}_{\mathcal{H}_{\varepsilon}^{0}}\left(\mathcal{A}_{\varepsilon}, \mathcal{A}_{0}\right)=0
$$

We now prove the existence of a Lyapunov functional for the semigroup $S_{\varepsilon}(t)$. This is a function $L \in C\left(\mathcal{H}_{\varepsilon}^{0}, \mathcal{H}_{\varepsilon}^{0}\right)$ satisfying the following properties:

(i) $L\left(S_{\varepsilon}(t) z\right) \leq L(z)$, for every $z \in \mathcal{H}_{\varepsilon}^{0}$;

(ii) $L\left(S_{\varepsilon}(t) z\right)=L(z)$ for every $t \geq 0$ implies that $z$ is a stationary point for $S_{\varepsilon}(t)$.

REMARK 13.3. The set of stationary points of $S_{\varepsilon}(t)$ is

$$
\mathbb{S}=\left\{\left(u^{\star}, 0,0\right): u^{\star} \in \mathcal{S}\right\}
$$

where $\mathcal{S}$ is the set of stationary points of (1.1) endowed with homogeneous Dirichlet boundary conditions.

As in the proof of Lemma 7.2 , we set

$$
\Phi(u)=\int_{0}^{u} \phi(y) d y .
$$

Proposition 13.4. For every $\varepsilon \in\left[0, \varepsilon_{0}\right]$,

$$
L(z)=\|z\|_{\mathcal{H}_{\varepsilon}^{0}}^{2}+2 \beta\langle\Phi(u), 1\rangle,
$$

with $z=(u, v, \eta) \in \mathcal{H}_{\varepsilon}^{0}$, is a Lyapunov functional for $S_{\varepsilon}(t)$.

Proof. It is clear that $L \in C\left(\mathcal{H}_{\varepsilon}^{0}, \mathcal{H}_{\varepsilon}^{0}\right)$. Besides, on account of (17.2), $L$ is nonincreasing along the trajectories of $S_{\varepsilon}(t)$. This proves (i). In order to show (ii), we observe that, again using (7.2), the equality $L\left(S_{\varepsilon}(t) z\right)=L(z)$ for every $t \geq 0$ implies that $\partial_{t} u(t)=0$ and $\eta^{t}=0$. In which case, the first equation of (6.3) reads

$$
-\Delta u+\phi(u)=0 \text {. }
$$


Hence, $S_{\varepsilon}(t) z=z$ for every $t \geq 0$.

The existence of a Lyapunov function ensures that $\mathcal{A}_{\varepsilon}$ coincides with the unstable manifold of $\mathbb{S}$ (which, in particular, is compact and nonempty), and that the next result holds (see [4, 23]).

TheOREM 13.5. For every $\varepsilon \in\left[0, \varepsilon_{0}\right]$ and every $z \in \mathcal{H}_{\varepsilon}^{0}$,

$$
\lim _{t \rightarrow \infty}\left[\inf _{z^{\star} \in \mathbb{S}}\left\|S_{\varepsilon}(t) z-z^{\star}\right\|_{\mathcal{H}_{\varepsilon}^{0}}\right]=0 .
$$

Thus, if $\varepsilon>0$,

$$
\lim _{t \rightarrow \infty}\left[\left\|\partial_{t} u(t)\right\|+\left\|\eta^{t}\right\|_{\mathcal{M}_{\varepsilon}^{0}}\right]=0
$$

Rephrasing the theorem, the $\omega$-limit set of $z$ belongs to $\mathbb{S}$, for every $z \in \mathcal{H}_{\varepsilon}^{0}$. In fact, setting $\iota=\lim _{t \rightarrow \infty} L\left(S_{\varepsilon}(t) z\right)$, it easily follows that

$$
\omega(z) \subset \mathbb{S}_{\iota}=\left\{z^{\star} \in \mathbb{S}: L\left(z^{\star}\right)=\iota\right\} .
$$

In particular, we have

Corollary 13.6. If $\mathbb{S}_{\iota}$ is discrete, there is $z^{\star} \in \mathbb{S}_{\iota}$ such that $S_{\varepsilon}(t) z \rightarrow z^{\star}$ in $\mathcal{H}_{\varepsilon}^{0}$ as $t \rightarrow \infty$.

On the other hand, in dimension two, $\mathbb{S}_{\iota}$ can be a continuum (e.g., if $\Phi$ is a doublewell potential [25]). Hence, the convergence of a given trajectory to a single equilibrium cannot be predicted, and it is false in general. Nonetheless, if $\phi$ is real analytic, there is a well-known tool which can be used in order to guarantee the convergence of trajectories to single stationary states: the Lojasiewicz-Simon inequality. If we consider the functional $E: H^{1} \rightarrow \mathbb{R}$, defined by

$$
E(u)=\frac{1}{2}\|\nabla u\|^{2}+\langle\Phi(u), 1\rangle,
$$

we have the following version of the Łojasiewicz-Simon inequality, devised by Haraux and Jendoubi [26].

Theorem 13.7. Let $\phi$ be real analytic, and let $u^{\star} \in \mathcal{S}$. Then, there exist $\theta=\theta\left(u^{\star}\right)>0$ and $\varsigma=\varsigma\left(u^{\star}\right)>0$ such that

$$
\left|E(u)-E\left(u^{\star}\right)\right|^{\frac{1+\theta}{1+2 \theta}} \leq\|\Delta u-\phi(u)\|_{H^{-1}},
$$

whenever $u \in H^{1}$ fulfills $\left\|\nabla u-\nabla u^{\star}\right\|<\varsigma$.

Thanks to this inequality, we can state and prove

Theorem 13.8. Let $\phi$ be real analytic. For every fixed $\varepsilon \in\left[0, \varepsilon_{0}\right]$ and every fixed $z \in \mathcal{H}_{\varepsilon}^{0}$, there exists $z^{\star}=\left(u^{\star}, 0,0\right) \in \mathbb{S}$ such that $\omega(z)=\left\{z^{\star}\right\}$. Moreover,

$$
\left\|S_{\varepsilon}(t) z-z^{\star}\right\|_{\mathcal{H}_{\varepsilon}^{0}} \leq \frac{c}{t^{\theta}},
$$

for some $\theta=\theta\left(u^{\star}\right)>0$ and $c=c(\varepsilon, z) \geq 0$.

We will restrict to $\varepsilon \in\left(0, \varepsilon_{0}\right]$. The (easier) case $\varepsilon=0$, besides having been treated by many authors (cf. 41] and references therein), can be easily recovered with minor changes in the proof by setting $\varepsilon=0$. We begin with a weaker result. 
LEMmA 13.9. The conclusion of Theorem 13.8 holds, with (13.2) replaced by the weaker estimate

$$
\left\|u(t)-u^{\star}\right\| \leq \frac{c}{t^{\theta}} .
$$

Proof. Let $z^{\star}=\left(u^{\star}, 0,0\right) \in \omega(z)$. In this proof, $c \geq 0$ will denote a generic constant, which may depend on $\varepsilon$ and $z$. In order to show that $\omega(z)=\left\{z^{\star}\right\}$, we follow the strategy of [26] (see also [5, 12]). We introduce the functional

$$
\Gamma(t)=2 \beta\left[E(u(t))-E\left(u^{\star}\right)\right]+\varepsilon\left\|\partial_{t} u(t)\right\|^{2}+\left\|\eta^{t}\right\|_{\mathcal{M}_{\varepsilon}^{0}}^{2}-2 \omega \varepsilon\left\langle\Delta u(t)-\phi(u(t)), \partial_{t} u(t)\right\rangle_{H^{-1}},
$$

for $\omega \in\left(0, \sigma_{0} / 2\right)$ to be chosen later, which fulfills the inequality

$$
\begin{aligned}
& \frac{d}{d t} \Gamma+\sigma_{0}\left\|\partial_{t} u\right\|^{2}+\frac{\delta}{\varepsilon}\|\eta\|_{\mathcal{M}_{\varepsilon}^{0}}^{2}+2 \omega \beta\|\Delta u-\phi(u)\|_{H^{-1}}^{2} \\
& \leq 2 \omega \varepsilon\left\langle\partial_{t} u, \phi^{\prime}(u) \partial_{t} u\right\rangle_{H^{-1}}+2 \omega\left\langle\sigma_{\varepsilon}(u) \partial_{t} u, \Delta u-\phi(u)\right\rangle_{H^{-1}}+2 \omega\langle\eta, \Delta u-\phi(u)\rangle_{\mathcal{M}_{\varepsilon}^{-1}} .
\end{aligned}
$$

Thanks to Lemma 7.2 provided that we fix $\omega$ small enough, the terms on the right-hand side are estimated by

$$
\begin{aligned}
2 \omega \varepsilon\left\langle\partial_{t} u, \phi^{\prime}(u) \partial_{t} u\right\rangle_{H^{-1}} & \leq \frac{\sigma_{0}}{4}\left\|\partial_{t} u\right\|^{2} \\
2 \omega\left\langle\sigma_{\varepsilon}(u) \partial_{t} u, \Delta u-\phi(u)\right\rangle_{H^{-1}} & \leq \frac{\sigma_{0}}{4}\left\|\partial_{t} u\right\|^{2}+\frac{\omega \beta}{2}\|\Delta u-\phi(u)\|_{H^{-1}}^{2}, \\
2 \omega\langle\eta, \Delta u-\phi(u)\rangle_{\mathcal{M}_{\varepsilon}^{-1}} & \leq \frac{\delta}{2 \varepsilon}\|\eta\|_{\mathcal{M}_{\varepsilon}^{0}}^{2}+\frac{\omega \beta}{2}\|\Delta u-\phi(u)\|_{H^{-1}}^{2} .
\end{aligned}
$$

Therefore, we obtain

$$
\frac{d}{d t} \Gamma+\rho_{0}\left(\left\|\partial_{t} u\right\|+\|\eta\|_{\mathcal{M}_{\varepsilon}^{0}}+\|\Delta u-\phi(u)\|_{H^{-1}}\right)^{2} \leq 0
$$

for some $\rho_{0}>0$. In particular, $\Gamma$ is a decreasing function, and so it has a limit (possibly not finite) when $t \rightarrow \infty$. On the other hand, since $z^{\star} \in \omega(z)$, we know that

$$
\lim _{n \rightarrow \infty} S_{\varepsilon}\left(t_{n}\right) z=z^{\star}
$$

for some $t_{n} \rightarrow \infty$. In turn,

$$
\lim _{t \rightarrow \infty} \Gamma(t)=\lim _{n \rightarrow \infty} \Gamma\left(t_{n}\right)=0 .
$$

We conclude that $\Gamma(t) \geq 0$. We now choose $\theta$ and $\varsigma$ as in Theorem 13.7. For $t$ large enough such that $\left\|\partial_{t} u(t)\right\| \leq 1$ and $\left\|\eta^{t}\right\|_{\mathcal{M}_{\varepsilon}^{0}} \leq 1$, from the very definition of $\Gamma$, we have

$$
\Gamma^{\frac{1+\theta}{1+2 \theta}} \leq c\left(\left|E(u)-E\left(u^{\star}\right)\right|^{\frac{1+\theta}{1+2 \theta}}+\left\|\partial_{t} u\right\|+\|\eta\|_{\mathcal{M}_{\varepsilon}^{0}}+\|\Delta u-\phi(u)\|_{H^{-1}}\right) .
$$

Besides, there is $m=m(\varsigma) \in \mathbb{N}$ such that

$$
\left\|\nabla u\left(t_{n}\right)-\nabla u^{\star}\right\|<\varsigma, \quad \forall n \geq m,
$$

and the inequalities $\left\|\partial_{t} u(t)\right\| \leq 1$ and $\left\|\eta^{t}\right\|_{\mathcal{M}_{\varepsilon}^{0}} \leq 1$ are satisfied for all $t \geq t_{m}$. Defining then, for every $n \geq m$,

$$
t_{n}^{\star}=\sup \left\{t \geq t_{n}:\left\|\nabla u(\tau)-\nabla u^{\star}\right\|<\varsigma, \forall \tau \in\left[t_{n}, t\right]\right\},
$$

we can apply Theorem 13.7, thus obtaining, for every $n \geq m$,

$$
\Gamma^{\frac{1+\theta}{1+2 \theta}} \leq c\left(\left\|\partial_{t} u\right\|+\|\eta\|_{\mathcal{M}_{\varepsilon}^{0}}+\|\Delta u-\phi(u)\|_{H^{-1}}\right), \quad \forall t \in\left[t_{n}, t_{n}^{\star}\right),
$$


for some $c$ independent of $n$. Combining (13.3)-(13.4), we deduce that

$$
\left\|\partial_{t} u\right\| \leq-c \Gamma^{-\frac{1+\theta}{1+2 \theta}} \frac{d}{d t} \Gamma=-\frac{c(1+2 \theta)}{\theta} \frac{d}{d t} \Gamma^{\frac{\theta}{1+2 \theta}},
$$

for all $t \in\left[t_{n}, t_{n}^{\star}\right)$ and every $n \geq m$. On the other hand, since $\Gamma(t) \rightarrow 0$ as $t \rightarrow \infty$, an integration of (13.5) yields

$$
\lim _{n \rightarrow \infty} \int_{t_{n}}^{t_{n}^{\star}}\left\|\partial_{t} u(\tau)\right\| d \tau=0 .
$$

The next step is to show that $t_{n}^{\star}=\infty$ for some $n$. If not,

$$
\lim _{n \rightarrow \infty}\left\|u\left(t_{n}^{\star}\right)-u^{\star}\right\| \leq \lim _{n \rightarrow \infty}\left(\left\|u\left(t_{n}\right)-u^{\star}\right\|+\int_{t_{n}}^{t_{n}^{\star}}\left\|\partial_{t} u(\tau)\right\| d \tau\right)=0 .
$$

But the existence of the global attractor implies that $u\left(t_{n}^{\star}\right)$ is precompact in $H^{1}$. As a consequence, $u\left(t_{n}^{\star}\right) \rightarrow u^{\star}$ in $H^{1}$, which contradicts the definition of $t_{n}^{\star}$. Thus, $t_{n}^{\star}=\infty$ for some $n$, and so $\partial_{t} u \in L^{1}\left(t_{n}, \infty ; H^{0}\right)$. This fact, together with the convergence $u\left(t_{n}\right) \rightarrow u^{\star}$, imply that $u(t) \rightarrow u^{\star}$ in $H^{0}$ as $t \rightarrow \infty$. Recalling (13.1), this proves that $\omega(z)=\left\{z^{\star}\right\}$. Finally, from (13.3) and (13.4),

$$
\frac{d}{d t} \Gamma+\rho_{1} \Gamma^{\frac{2(1+\theta)}{1+2 \theta}} \leq 0, \quad \forall t \geq t_{n}
$$

for some $\rho_{1}>0$. Hence,

$$
\Gamma(t) \leq \frac{c}{t^{1+2 \theta}}, \quad \forall t \geq t_{n},
$$

which, by integrating (13.5), entails

$$
\left\|u(t)-u^{\star}\right\| \leq \int_{t}^{\infty}\left\|\partial_{t} u(\tau)\right\| d \tau \leq \frac{c}{t^{\theta}}, \quad \forall t \geq t_{n} .
$$

Clearly, the inequality holds for every $t>0$.

Proof of Theorem 13.8. In order to prove (13.2), we use the trajectory decomposition of Section 10, namely,

$$
S_{\varepsilon}(t) z=S_{\varepsilon}^{1}(t) z+S_{\varepsilon}^{2}(t) z=\left(v(t), \partial_{t} v(t), \psi^{t}\right)+\left(w(t), \partial_{t} w(t), \xi^{t}\right) .
$$

Then, in view of (10.1), we are left to prove that

$$
\left\|\nabla w(t)-\nabla u^{\star}\right\|+\sqrt{\varepsilon}\left\|\partial_{t} w(t)\right\|+\left\|\xi^{t}\right\|_{\mathcal{M}_{\varepsilon}^{0}} \leq \frac{c}{t^{\theta}} .
$$

Setting $w^{\star}=w-u^{\star}$, we define

$$
\Xi(t)=\beta\left\|\nabla w^{\star}(t)\right\|^{2}+\varepsilon\left\|\partial_{t} w(t)\right\|^{2}+\left\|\xi^{t}\right\|_{\mathcal{M}_{\varepsilon}^{0}}^{2}+2 \omega \varepsilon\left\langle w^{\star}(t), \partial_{t} w(t)\right\rangle,
$$

with $\omega \in\left(0, \sigma_{0} / 2\right)$ small enough such that

$$
\frac{1}{2}\left\|\left(w^{\star}(t), \partial_{t} w(t), \xi^{t}\right)\right\|_{\mathcal{H}_{\varepsilon}^{0}}^{2} \leq \Xi(t) \leq 2\left\|\left(w^{\star}(t), \partial_{t} w(t), \xi^{t}\right)\right\|_{\mathcal{H}_{\varepsilon}^{0}}^{2} .
$$


Multiplying the first equation of (10.4) by $\partial_{t} w+\omega w^{\star}$ in $H^{0}$ and the second one by $\xi$ in $\mathcal{M}_{\varepsilon}^{0}$, we are led to

$$
\begin{aligned}
\frac{d}{d t} \Xi+2 \omega \beta\left\|\nabla w^{\star}\right\|^{2}+\sigma_{0}\left\|\partial_{t} w\right\|^{2}+\frac{\delta}{\varepsilon}\|\xi\|_{\mathcal{M}_{\varepsilon}^{0}}^{2} \\
\leq-2 \beta\left\langle\phi(w)-\phi\left(u^{\star}\right), \partial_{t} w\right\rangle+2 \varpi \beta\left\langle v, \partial_{t} w\right\rangle-2 \omega\left\langle\sigma_{\varepsilon}(w) \partial_{t} w, w^{\star}\right\rangle \\
\quad-2 \omega\left\langle\xi, w^{\star}\right\rangle_{\mathcal{M}_{\varepsilon}^{0}}-2 \omega \beta\left\langle\phi(w)-\phi\left(u^{\star}\right), w^{\star}\right\rangle+2 \omega \varpi \beta\left\langle v, w^{\star}\right\rangle .
\end{aligned}
$$

Exploiting (10.2), the right-hand side is estimated by standard computations, yielding

$$
\frac{d}{d t} \Xi+\rho_{2} \Xi \leq c\left(\left\|w^{\star}\right\|^{2}+\|v\|^{2}\right) \leq \frac{c}{t^{2 \theta}},
$$

for some $\rho_{2}>0$, where the last inequality follows from the obvious estimate

$$
\left\|w^{\star}\right\|^{2} \leq 2\left\|u-u^{\star}\right\|^{2}+2\|v\|^{2},
$$

along with (10.1) and Lemma 13.9. The Gronwall lemma entails the desired conclusion.

14. Conclusion: Proofs of the main results. Theorem 6.3, along with Corollary 6.2, ensure the existence and uniqueness of a solution to (5.1). In turn, Proposition 5.3 provides the existence of a solution to (1.4), whose uniqueness is established by Proposition 4.2. This completes the proof of Proposition 3.2 and Proposition 3.4. In particular, we find the relationship

$$
U_{\varepsilon}(t) u_{0}=\mathbb{P} S_{\varepsilon}(t)\left(u_{0},-\phi\left(u_{0}\right), u_{0}\right),
$$

where, here and in the sequel, $\mathbb{P}: \mathcal{H}_{\varepsilon}^{0} \rightarrow H^{1}$, defined as

$$
\mathbb{P}\left(u_{0}, v_{0}, \eta_{0}\right)=u_{0},
$$

is the projection of $\mathcal{H}_{\varepsilon}^{0}$ onto its first component $H^{1}$.

Proof of Theorem 3.5. The idea is to make use of the estimate (11.1) provided by Theorem 11.1. Unfortunately, this cannot be done directly, since $u_{0}$ does not belong to $\operatorname{dom}\left(T_{\varepsilon}\right)$ (unless it is zero). This obstacle can be circumvented as follows: if $\left\|\Delta u_{0}\right\| \leq R$, by Theorem 11.1, we learn that

$$
\left\|\nabla \mathbb{P} S_{\varepsilon}(t)\left(u_{0},-\phi\left(u_{0}\right), 0\right)-\nabla U_{0}(t) u_{0}\right\| \leq \mathcal{Q}(T+R) \varepsilon^{a} .
$$

Then, we observe that

$$
\mathbb{P} S_{\varepsilon}(t)\left(u_{0},-\phi\left(u_{0}\right), u_{0}\right)=\mathbb{P} S_{\varepsilon}^{f}(t)\left(u_{0},-\phi\left(u_{0}\right), 0\right),
$$

where we set

$$
f(t)=h(t / \varepsilon) \Delta u_{0}, \quad \text { with } \quad h(t)=\int_{t}^{\infty} \mu(s) d s .
$$

Indeed, by means of (6.2), the third component $\eta^{t}$ of $S_{\varepsilon}(t)\left(u_{0},-\phi\left(u_{0}\right), u_{0}\right)$ reads

$$
\eta^{t}(s)=\tilde{\eta}^{t}(s)+ \begin{cases}0, & 0<s \leq t, \\ u_{0}, & s>t,\end{cases}
$$


where $\tilde{\eta}^{t}$ is the mild solution to the Cauchy problem

$$
\left\{\begin{array}{l}
\partial_{t} \tilde{\eta}^{t}=T_{\varepsilon} \tilde{\eta}^{t}+\partial_{t} u(t), \quad t>0 \\
\tilde{\eta}^{0}=0
\end{array}\right.
$$

Accordingly,

$$
\int_{0}^{\infty} \mu_{\varepsilon}(s) \Delta \eta^{t}(s) d s=\int_{0}^{\infty} \mu_{\varepsilon}(s) \Delta \tilde{\eta}^{t}(s) d s+f(t),
$$

which proves the claim. At this point, we can apply Proposition 9.1, so obtaining

$$
\begin{aligned}
& \left\|\nabla \mathbb{P} S_{\varepsilon}(t)\left(u_{0},-\phi\left(u_{0}\right), 0\right)-\nabla U_{\varepsilon}(t) u_{0}\right\| \\
& \leq\left\|S_{\varepsilon}(t)\left(u_{0},-\phi\left(u_{0}\right), 0\right)-S_{\varepsilon}^{f}(t)\left(u_{0},-\phi\left(u_{0}\right), 0\right)\right\|_{\mathcal{H}_{\varepsilon}^{0}} \\
& \leq \mathcal{Q}(t+R)\left(\int_{0}^{t}[h(\tau / \varepsilon)]^{2} d \tau\right)^{\frac{1}{2}} \\
& \leq \mathcal{Q}(T+R) \sqrt{\varepsilon} .
\end{aligned}
$$

Collecting (14.1) and (14.2), we are done.

Proof of Theorem 3.6. Due to Proposition 7.1, the set $\mathcal{B}^{1}=\bigcup_{\varepsilon \in\left[0, \varepsilon_{0}\right]} \mathbb{P B}_{\varepsilon}^{0}$ is bounded in $H^{1}$. Moreover, given a bounded set $\mathcal{B} \subset H^{1}$, we have

$$
U_{\varepsilon}(t) \mathcal{B}=\mathbb{P} S_{\varepsilon}(t) \mathbb{B} \subset \mathbb{P B}_{\varepsilon}^{0} \subset \mathcal{B}^{1},
$$

for every $t \geq t_{0}\left(\|\mathbb{B}\|_{\mathcal{H}_{\varepsilon}^{0}}\right)=t_{0}\left(\|\mathcal{B}\|_{H^{1}}\right)$, where

$$
\mathbb{B}=\left\{\left(u_{0},-\phi\left(u_{0}\right), u_{0}\right): u_{0} \in \mathcal{B}\right\} .
$$

The desired $\mathcal{B}^{0}$ is obtained by setting

$$
\mathcal{B}^{0}=\bigcup_{t \in\left[0, t_{1}\right]} U_{\varepsilon}(t) \mathcal{B}^{1},
$$

with $t_{1}=t_{0}\left(\left\|\mathcal{B}^{1}\right\|_{H^{1}}\right)$. By Lemma $7.2, \mathcal{B}^{0}$ is bounded in $H^{1}$.

Proof of Theorem 3.7. Define $\mathcal{K}_{\varepsilon}=\mathbb{P} \mathcal{E}_{\varepsilon}$. Then, $\mathcal{K}_{\varepsilon}$ is compact in $H^{1}$ and bounded in $H^{2}$. Besides, thanks to point (I) of Theorem 12.1 ,

$$
\operatorname{dim}_{\text {frac }}\left(\mathcal{K}_{\varepsilon} ; H^{1}\right) \leq \operatorname{dim}_{\text {frac }}\left(\mathcal{E}_{\varepsilon} ; \mathcal{H}_{\varepsilon}^{0}\right) \leq C .
$$

Let now $\mathcal{B} \subset H^{1}$ be a bounded set. Setting $\mathbb{B}$ as in the previous proof, and appealing to point (II) of Theorem 12.1, we have

$$
\begin{aligned}
\boldsymbol{\delta}_{H^{1}}\left(U_{\varepsilon}(t) \mathcal{B}, \mathcal{K}_{\varepsilon}\right) & =\boldsymbol{\delta}_{H^{1}}\left(\mathbb{P} S_{\varepsilon}(t) \mathbb{B}, \mathbb{P} \mathcal{E}_{\varepsilon}\right) \\
& \leq \boldsymbol{\delta}_{\mathcal{H}_{\varepsilon}^{0}}\left(S_{\varepsilon}(t) \mathbb{B}, \mathcal{E}_{\varepsilon}\right) \\
& \leq \mathcal{Q}\left(\|\mathbb{B}\|_{\mathcal{H}_{\varepsilon}^{0}}\right) e^{-\kappa t} \\
& =\mathcal{Q}\left(\|\mathcal{B}\|_{H^{1}}\right) e^{-\kappa t} .
\end{aligned}
$$

Finally, exploiting point (III), we learn that

$$
\boldsymbol{\delta}_{H^{1}}^{\text {sym }}\left(\mathcal{K}_{\varepsilon}, \mathcal{K}_{0}\right)=\boldsymbol{\delta}_{H^{1}}^{\text {sym }}\left(\mathbb{P} \mathcal{E}_{\varepsilon}, \mathbb{P} \mathcal{E}_{0}\right) \leq \boldsymbol{\delta}_{\mathcal{H}_{\varepsilon}^{0}}^{\text {sym }}\left(\mathcal{E}_{\varepsilon}, \mathcal{E}_{0}\right) \leq C \varepsilon^{\tau},
$$

which completes the proof.

Proofs of Theorem 3.8 and Theorem 3.10. Apply Theorem 13.5 and Theorem 13.8 to the vectors $z=\left(u_{0},-\phi\left(u_{0}\right), u_{0}\right)$. 


\section{REFERENCES}

[1] S. Aizicovici, M. Grasselli, M. McKibben, A hyperbolic integrodifferential system related to phasefield models, Adv. Math. Sci. Appl. 10, 601-625, 2000. MR1807443 (2002h:45013)

[2] A. Babin, M.I. Vishik, Attractors of evolution equations, North-Holland, Amsterdam, 1992. MR.1156492 (93d:58090)

[3] V. Belleri, V. Pata, Attractors for semilinear strongly damped wave equation on $\mathbb{R}^{3}$, Discrete Contin. Dynam. Systems 7, 719-735, 2001. MR.1849655 (2003f:35026)

[4] T. Cazenave, A. Haraux, An introduction to semilinear evolution equations, Oxford University Press, New York, 1998. MR.1691574 (2000e:35003)

[5] R. Chill, E. Fašangová, Convergence to steady states of solutions of semilinear evolutionary integral equations, Calc. Var. Partial Differential Equations 22, 321-342, 2005. MR2118902 (2005i:45013)

[6] M. Conti, V. Pata, M. Squassina, Singular limit of differential systems with memory, Indiana Univ. Math. J. 55, 169-215, 2006. MR2207550 (2006k:35290)

[7] C.M. Dafermos, Asymptotic stability in viscoelasticity, Arch. Rational Mech. Anal. 37, 554-569, 1970. MR0281400(43:7117)

[8] B.R. Duffy, P. Freitas, M. Grinfeld, Memory driven instability in a diffusion process, SIAM J. Math. Anal. 33, 1090-1106, 2002. MR.1897703 (2003a:76008)

[9] P. Fabrie, C. Galusinski, A. Miranville, S. Zelik, Uniform exponential attractors for a singularly perturbed damped wave equation, Discrete Contin. Dynam. Systems 10, 211-238, 2004. MR2026192 (2006c:37088)

[10] J. Fort, V. Méndez, Wavefront in time-delayed reaction-diffusion systems. Theory and comparison to experiments, Rep. Prog. Phys. 65, 895-954, 2002.

[11] P. Freitas, Eigenvalue problems for the wave equation with strong damping, Proc. Roy. Soc. Edinburgh Sect. A 127A, 755-771, 1997. MR1465418 (98g:35133)

[12] S. Gatti, M. Grasselli, Convergence to stationary states of solutions to the semilinear equation of viscoelasticity, in "Differential Equations: Inverse and Direct Problems" (A. Favini and A. Lorenzi, Eds.), pp. 131-147, Ser. Lect. Notes Pure Appl. Math. no. 251, Chapman \& Hall/CRC, Boca Raton, 2006. MR2275976 (2007g:35249)

[13] S. Gatti, M. Grasselli, A. Miranville, V. Pata, Memory relaxation of first order evolution equations, Nonlinearity, 18, 1859-1883, 2005. MR2150358 (2006e:35209)

[14] S. Gatti, M. Grasselli, A. Miranville, V. Pata, A construction of a robust family of exponential attractors, Proc. Amer. Math. Soc. 134, 117-127, 2006. MR2170551(2006e:37143)

[15] S. Gatti, V. Pata, A one-dimensional wave equation with nonlinear damping, Glasg. Math. J. 48, 419-430, 2006. MR.2271373 (2007k:35331)

[16] G. Gentili and C. Giorgi, Thermodynamic properties and stability for the heat flux equation with linear memory, Quart. Appl. Math. 51, 342-362, 1993. MR.1218373 (94j:80004)

[17] C. Giorgi, A. Marzocchi, V. Pata, Asymptotic behavior of a semilinear problem in heat conduction with memory, NoDEA Nonlinear Differential Equations Appl. 5, 333-354, 1998. MR.1638908 (99h:35081)

[18] C. Giorgi, V. Pata, Asymptotic behavior of a nonlinear hyperbolic heat equation with memory, NoDEA Nonlinear Differential Equations Appl. 8, 157-171, 2001. MR1834457 (2002d:35210)

[19] M. Grasselli, V. Pata, Upper semicontinuous attractor for a hyperbolic phase-field model with memory, Indiana University Mathematics Journal 50, 1281-1308, 2001. MR1871356 (2002h:35031)

[20] M. Grasselli, V. Pata, Uniform attractors of nonautonomous systems with memory, in "Evolution Equations, Semigroups and Functional Analysis" (A. Lorenzi and B. Ruf, Eds.), pp. 155-178, Progr. Nonlinear Differential Equations, Appl. no.50, Birkhäuser, Boston, 2002. MR.1944162 (2003j:37135)

[21] M. Grasselli, V. Pata, A reaction-diffusion equation with memory, Discrete Contin. Dyn. Syst. 15, 1079-1088, 2006. MR2224498 (2006m:35184)

[22] M.E. Gurtin, A.C. Pipkin, A general theory of heat conduction with finite wave speeds, Arch. Rational Mech. Anal. 31, 113-126, 1968. MR.1553521

[23] J.K. Hale, Asymptotic behaviour of dissipative systems, Amer. Math. Soc., Providence, RI, 1988. MR941371 (89g:58059)

[24] J.K. Hale, G. Raugel, Upper semicontinuity of the attractor for a singularly perturbed hyperbolic equation, J. Differential Equations 73, 197-214, 1988. MR943939 (89i:35022)

[25] A. Haraux, Systèmes dynamiques dissipatifs et applications, Masson, Paris, 1991. MR1084372 (92b:35002) 
[26] A. Haraux, M.A. Jendoubi, Convergence of bounded weak solutions of the wave equation with dissipation and analytic nonlinearity, Calc. Var. Partial Differential Equations 9, 95-124, 1999. MR 1714129 (2000h:35110)

[27] D.B. Henry, Geometric theory of semilinear parabolic equations, Springer-Verlag, New York, 1981. MR610244 (83j:35084)

[28] T. Hillen, Qualitative analysis of semilinear Cattaneo equations, Math. Models Methods Appl. Sci. 8, 507-519, 1998. MR1624816 (99c:35121)

[29] T. Hillen, K.P. Hadeler, Hyperbolic systems and transport equations in mathematical biology, in "Analysis and numerics for conservation laws", pp. 257-279, Springer, Berlin, 2005. MR2169931 (2006e:92024)

[30] N. Kopell, D. Ruelle, Bounds on complexity in reaction-diffusion systems, SIAM J. Appl. Math. 46, 68-80, 1986. MR $821442(87 \mathrm{~d}: 35020)$

[31] M. Marion, Attractors for reaction-diffusion equations: Existence and estimates of their dimensions, Appl. Analysis 25, 101-147, 1987. MR911962 (88m:35082)

[32] V. Méndez, J. Fort, J. Farjas, Speed of wavefront solutions to hyperbolic reaction-diffusion systems, Phys. Rev. E 60, 5231-5243, 1999. MR1719397 (2000h:35094)

[33] V. Méndez, J.E. Llebot, Hyperbolic reaction-diffusion equations for a forest fire model, Phys. Rev. E 56, 6557-6563, 1997. MR 1492398

[34] J.W. Nunziato, On heat conduction in materials with memory, Quart. Appl. Math. 29, 187-204, 1971. MR0295683 (45:4749)

[35] W.E. Olmstead, S.H. Davis, S. Rosenblat, W.L. Kath, Bifurcation with memory, SIAM J. Appl. Math. 46, 171-188, 1986. MR833472 (87f:35020)

[36] V. Pata, S. Zelik, Attractors and their regularity for 2-D wave equation with nonlinear damping, Adv. Math. Sci. Appl. 17, 225-237, 2007. MR2337377 (2008j:37168)

[37] V. Pata, S. Zelik, Global and exponential attractors for 3-D wave equations with displacement dependent damping, Math. Methods Appl. Sci. 29, 1291-1306, 2006. MR2247700 (2007h:37127)

[38] V. Pata, A. Zucchi, Attractors for a damped hyperbolic equation with linear memory, Adv. Math. Sci. Appl. 11, 505-529, 2001. MR.1907454 (2003f:35027)

[39] A. Pazy, Semigroups of linear operators and applications to partial differential equations, SpringerVerlag, New York, 1983. MR710486 (85g:47061)

[40] R. Temam, Infinite-dimensional dynamical systems in mechanics and physics, Springer, New York, 1988. MR 953967 (89m:58056)

[41] S. Zheng, Nonlinear evolution equations, Chapman \& Hall/CRC, Boca Raton, 2004. MR2088362 (2006a:35001) 